\title{
Detection of a hot core in the intermediate-mass Class 0 protostar NGC 7129-FIRS 2
}

\author{
A. Fuente ${ }^{1}$, R. Neri ${ }^{2}$, and P. Caselli ${ }^{3}$ \\ 1 Observatorio Astronómico Nacional (IGN), Campus Universitario, Apdo. 112, 28803 Alcalá de Henares (Madrid), Spain \\ e-mail: a.fuente@oan.es \\ 2 Institut de Radioastronomie Millimétrique, 300 rue de la Piscine, 38406 St. Martin d'Hères Cedex, France \\ 3 INAF - Osservatorio Astrofisico de Arcetri, Largo Enrico Fermi 5, 50125 Firenze, Italy
}

Received 25 April 2005 / Accepted 24 June 2005

\section{ABSTRACT}

We report high angular resolution $\left(H P B W \sim 0.6^{\prime \prime} \times 0.5^{\prime \prime}\right.$ at $\left.1.3 \mathrm{~mm}\right)$ observations of the Class 0 intermediate-mass (IM) protostar NGC 7129FIRS 2 using the Plateau de Bure Interferometer. Our observations show the existence of an intense unresolved source in the continuum at $1.3 \mathrm{~mm}$ and $3 \mathrm{~mm}$ at the position of the Class 0 object. In addition, compact $\mathrm{CH}_{3} \mathrm{CN}$ emission is detected at this position. The high rotational temperature derived from the $\mathrm{CH}_{3} \mathrm{CN}$ lines $\left(T_{\text {rot }} \approx 50 \mathrm{~K}\right)$, as well as the enhanced $\mathrm{CH}_{3} \mathrm{CN}$ fractional abundance $\left(X\left(\mathrm{CH}_{3} \mathrm{CN}\right) \sim 7.0 \times 10^{-9}\right)$, shows the existence of a hot core in this IM young stellar object. This is to our knowledge the first IM hot core detected so far. Interferometric maps of the region in the $\mathrm{CH}_{3} \mathrm{OH} 5_{k k^{\prime}} \rightarrow 4_{k k^{\prime}}$ and $\mathrm{D}_{2} \mathrm{CO} 4_{04} \rightarrow 3_{03}$ lines are also presented in this paper. The methanol emission presents two condensations, one associated with the hot core, which was very intense in the high upper state energy lines $\left(E_{u}>100 \mathrm{~K}\right)$, and the other associated with the bipolar outflow which dominates the emission in the low excitation lines. Enhanced $\mathrm{CH}_{3} \mathrm{OH}$ abundances $\left(X\left(\mathrm{CH}_{3} \mathrm{OH}\right) \sim 3 \times 10^{-8}-\mathrm{a}\right.$ few $\left.10^{-7}\right)$ were measured in both components. While intense $\mathrm{D}_{2} \mathrm{CO} 4_{04} \rightarrow 3_{03}$ emission was detected towards the hot core, the $\mathrm{N}_{2} \mathrm{D}^{+} 3 \rightarrow 2$ line was not detected in our interferometric observations. The different behaviors of $\mathrm{D}_{2} \mathrm{CO}$ and $\mathrm{N}_{2} \mathrm{D}^{+}$emissions suggest different formation mechanisms for the two species and different deuteration processes for $\mathrm{H}_{2} \mathrm{CO}$ and $\mathrm{N}_{2} \mathrm{H}^{+}$(surface and gas-phase chemistry, respectively). Finally, the spectrum of the large bandwidth correlator shows a forest of lines at the hot core position, revealing that this object is extraordinarily rich in complex molecules. For deeper insight into the chemistry of complex molecules, we compared the fractional abundances of the complex $\mathrm{O}$ - and $\mathrm{N}$ bearing species in FIRS 2 with those in hot corinos and massive hot cores. Within the large uncertainty involved in fractional abundance estimates towards hot cores, we did not detect any variation in the relative abundances of $\mathrm{O}$ - and $\mathrm{N}$-bearing molecules $\left(\left[\mathrm{CH} \mathrm{CN}_{3} /\left[\mathrm{CH} \mathrm{OH}_{3} \mathrm{OH}\right)\right.\right.$ with the hot core luminosity. However, the O-bearing species $\mathrm{H}_{2} \mathrm{CO}$ and $\mathrm{HCOOH}$ seemed to be more abundant in low and intermediate mass stars than in massive star-forming regions. We propose that this could be the consequence of a different grain mantle composition in low and massive star-forming regions.

Key words. stars: formation - stars: individual: NGC 7129-FIRS 2 - ISM: abundances - ISM: individual objects: NGC 7129

\section{Introduction}

Hot cores are compact objects near or around protostars characterized by warm temperatures $\left(T_{k}>100 \mathrm{~K}\right)$ and high densities $\left(n>10^{6} \mathrm{~cm}^{-3}\right)$. These regions are also characterized by a very rich chemistry in complex molecules $\left(\mathrm{CH}_{3} \mathrm{OH}\right.$, $\mathrm{CH}_{3} \mathrm{CN}, \mathrm{CH}_{3} \mathrm{OCHO}, \mathrm{CH}_{3} \mathrm{OCH}_{3}, \mathrm{C}_{2} \mathrm{H}_{5} \mathrm{CN}$...). Hot cores are thought to be associated with high-mass protostars $(M \geq$ $8 M_{\odot}$ ) and to represent an important phase in their evolution toward ultracompact and compact HII regions. Recently, regions characterized by warm temperatures and high densities have also been detected in two low-mass protostars IRAS 16293-2422 (Ceccarelli et al. 2000; Cazaux et al. 2003) and NGC 1333 IRAS 4A (Bottinelli et al. 2004). Complex molecules typical of hot cores (e.g. $\mathrm{HCOOH}, \mathrm{CH}_{3} \mathrm{OCHO}$, $\mathrm{CH}_{3} \mathrm{CN}, \mathrm{C}_{2} \mathrm{H}_{5} \mathrm{CN}$ ) have also been detected in these objects.
However, the amount of warm material involved, as well as the chemistry, are different in the two classes of objects. For this reason, the warm regions in the inner envelope of low mass protostars are usually referred to as "hot corinos".

The formation of complex molecules in hot cores and corinos is poorly understood. In the standard scheme, neutral molecules (CO, CS...) are frozen onto dust grains during the cold pre-stellar phase. If the dust temperature is sufficiently low during this phase, surface hydrogenation of $\mathrm{CO}$ leads to the formation of solid $\mathrm{H}_{2} \mathrm{CO}$ and $\mathrm{CH}_{3} \mathrm{OH}$ (e.g. Brown et al. 1988; Charnley et al. 1992; Caselli et al. 1993). Once the star starts heating the grain surfaces, these molecules (called "parent" species) evaporate, enlarging their abundances in the gas phase. Because of the high temperature and density of hot cores and corinos, these molecules undergo fast neutral-neutral and ionneutral reactions producing a second generation of complex 
O-bearing species called "daughter" molecules at early stages ( $\sim 10^{4} \mathrm{yr}$ ), such as methyl formate, $\mathrm{CH}_{3} \mathrm{OCHO}$. However, Horn et al. (2004) recently found that the gas-phase reaction sequence between protonated methanol and formaldehyde, crucial for the gas-phase formation of methyl formate, does not proceed in their laboratory experiments. This suggests that surface chemistry is probably also playing a key role in the formation of this species.

Complex N-bearing species (e.g. $\mathrm{CH}_{3} \mathrm{CN}, \mathrm{CH}_{3} \mathrm{CH}_{2} \mathrm{CN}$ ) are observed in hot cores and hot corinos. In the chemical scheme of Rodgers \& Charnley (2001), complex N-bearing species are thought to be formed in the gas phase about $10^{5} \mathrm{yr}$ after the grain mantle evaporation, but only if the gas temperature is sufficiently large ( $\geq 300 \mathrm{~K})$. On the other hand, Caselli et al. (1993) found that large abundances of ethyl cyanide $\left(X\left(\mathrm{CH}_{3} \mathrm{CH}_{2} \mathrm{CN}\right) \sim 10^{-7}\right.$, w.r.t. $\left.\mathrm{H}_{2}\right)$ can be obtained on grain surfaces, if the dust temperature during protostellar accreting is around $40 \mathrm{~K}$. In the case of lower temperatures $(10-20 \mathrm{~K})$, the $\mathrm{CH}_{3} \mathrm{CH}_{2} \mathrm{CN}$ can still be formed on the surface, but with reduced abundances $\left(\sim 10^{-9}\right)$. Thus, Caselli et al. (1993) suggest that saturated (or $\mathrm{H}$-rich) complex $\mathrm{N}$-bearing species (such as ethyl cyanide) are "parent" species and that they should be observable as soon as the grain mantles are released in the gas phase upon stellar heating of the dust. Later on, "H-poor" $\mathrm{N}$-bearing molecules (e.g. vinyl cyanide, $\mathrm{CH}_{2} \mathrm{CHCN}$ ) can be formed in the gas phase from the destruction of $\mathrm{CH}_{3} \mathrm{CH}_{2} \mathrm{CN}$.

Indeed, if complex species in general are not formed on grain surfaces, there are important problems for understanding the chemical composition of hot corinos: the timescale necessary to convert "parent" molecules into complex "daughter" molecules is much longer than the transit time of the gas in hot corinos (a few hundred years). Therefore, it seems that the chemistry of complex molecules must begin on grain surfaces (at least in hot corinos). However, chemists are still far from providing a unique interpretation of the hot core (and corino) chemistry, so, more observational work is needed. In particular, given the large sensitivity of surface and gas-phase chemistry on dust temperature and gas density, it will be extremely important to measure possible variations of complex molecule abundances with the physical characteristics of hot cores, which of course depend on the mass and luminosity of the associated protostar.

In this paper, we present an interferometric study of the hot core associated with the Class 0 IM protostar NGC 7129-FIRS 2 . With a luminosity $\sim 500 L_{\odot}$ and a mass $\sim 5 M_{\odot}$, FIRS 2 is very likely the youngest IM object known at present (Fuente et al. 1998, 2002). An energetic bipolar molecular outflow with a quadrupolar morphology is associated with it (Fuente et al. 2001, hereafter Paper I). Interferometric observations in the continuum at $1 \mathrm{~mm}$ and the ${ }^{12} \mathrm{CO} 2 \rightarrow 1$ line shows that the quadrupolar morphology of the outflow is due to the superposition of two bipolar molecular outflows FIRS 2-out 1 and FIRS 2-out 2. FIRS 2-out 1 is associated with the Class 0 protostar detected as an intense millimeter clump in the continuum image (MM1 in the nomenclature of Paper I), while FIRS 2-out 2 is associated with a more evolved infrared star (FIRS 2 - IR) undetected at millimeter wavelengths.
Table 1. Observations with the Plateau de Bure Interferometer (PdBI).

\begin{tabular}{lccc}
\hline \hline & Conf. & Beam & Date \\
\hline Continuum $3 \mathrm{~mm}$ & A & $1.56^{\prime \prime} \times 1.20^{\prime \prime}$ & March 03 \\
Continuum 1 mm & A & $0.63^{\prime \prime} \times 0.46^{\prime \prime}$ & March 03 \\
$\mathrm{CH}_{3} \mathrm{CN} 5_{k} \rightarrow 4_{k}$ & A & $1.56^{\prime \prime} \times 1.20^{\prime \prime}$ & March 03 \\
$\mathrm{N}_{2} \mathrm{D}^{+} 3 \rightarrow 2$ & A & $0.63^{\prime \prime} \times 0.46^{\prime \prime}$ & March 03 \\
$\mathrm{D}_{2} \mathrm{CO} 4_{04} \rightarrow 3_{03}$ & A & $0.63^{\prime \prime} \times 0.46^{\prime \prime}$ & March 03 \\
$\mathrm{CH}_{3} \mathrm{OH} 5_{k, k^{\prime}} \rightarrow 4_{k, k^{\prime}}$ & CD & $1.51^{\prime \prime} \times 1.42^{\prime \prime}$ & Nov., Dec. 98 \\
\hline
\end{tabular}

A quite complete chemical study of this Class 0 IM source was carried out by Fuente et al. (2005) (hereafter Paper II) using the $30 \mathrm{~m}$ IRAM telescope. They detected warm $\mathrm{CH}_{3} \mathrm{CN}$ $\left(T_{k}>63 \mathrm{~K}\right)$ towards the central position, which constitutes strong evidence of the existence of a hot core in this Class 0 IM object. However, the limited angular resolution of these observations makes it very difficult to distinguish between the hot core emission and those of the outflow and/or the warm envelope. The new results presented in this paper confirm the existence of the hot core associated with FIRS 2 and give a first glance at the chemistry of this object. To our knowledge, FIRS 2 is the first IM hot core detected thus far. The intermediate kinetic temperature and mass of the FIRS 2 hot core are expected to produce a differentiated chemistry and to furnish a link between the low mass and high mass regimes.

\section{Observations}

The observations presented in this paper correspond to two different observational projects carried out with the Plateau de Bure interferometer (PdBI). The main set of data was observed on March 08, 2003. The observations were carried out with excellent weather conditions and counts $5 \mathrm{hr}$ of integration time (on-source) with 6-element array in the A configuration, which provides the highest angular resolution. The spectral correlator setup was adjusted to observe the $\mathrm{CH}_{3} \mathrm{CN} 5 \rightarrow 4$ line with two contiguous $20 \mathrm{MHz}$ units, $\mathrm{N}_{2} \mathrm{D}^{+} 3 \rightarrow 2$ and $\mathrm{D}_{2} \mathrm{CO}$ $4_{04} \rightarrow 3_{03}$, with two separated $20 \mathrm{MHz}$ units; and the remaining units were configured for maximum continuum sensitivity (see Table 1). These observations show average system temperatures of $140 \mathrm{~K}(3 \mathrm{~mm})$ and $250 \mathrm{~K}(1 \mathrm{~mm})$, a mean atmospheric water vapour content of $1-2 \mathrm{~mm}$, and were made under seeing conditions of 0.3 arcsec. The RF calibrator was 0420 0414, the phase calibrators ordered by right ascension were $1928+738,2146+608$, and $2309+454$, and the flux calibrator was MWC 349. The precision in the flux densities is better than $20 \%$ at $1 \mathrm{~mm}$ and better than $10 \%$ at $3 \mathrm{~mm}$. Images were created in natural weighting.

In addition, we present the $\mathrm{CH}_{3} \mathrm{OH} J=5_{k} \rightarrow 4_{k}$ image observed during a previous project in November and December, 1998. The data corresponding to this project were partially published in Paper I. Details about the observations are given in Table 1 and Paper I.

\section{Continuum data}

The continuum images at $3 \mathrm{~mm}$ and $1.3 \mathrm{~mm}$ are shown in Fig. 1. The angular resolution of these images is 3 times better than for images previously published in Paper I. Both 


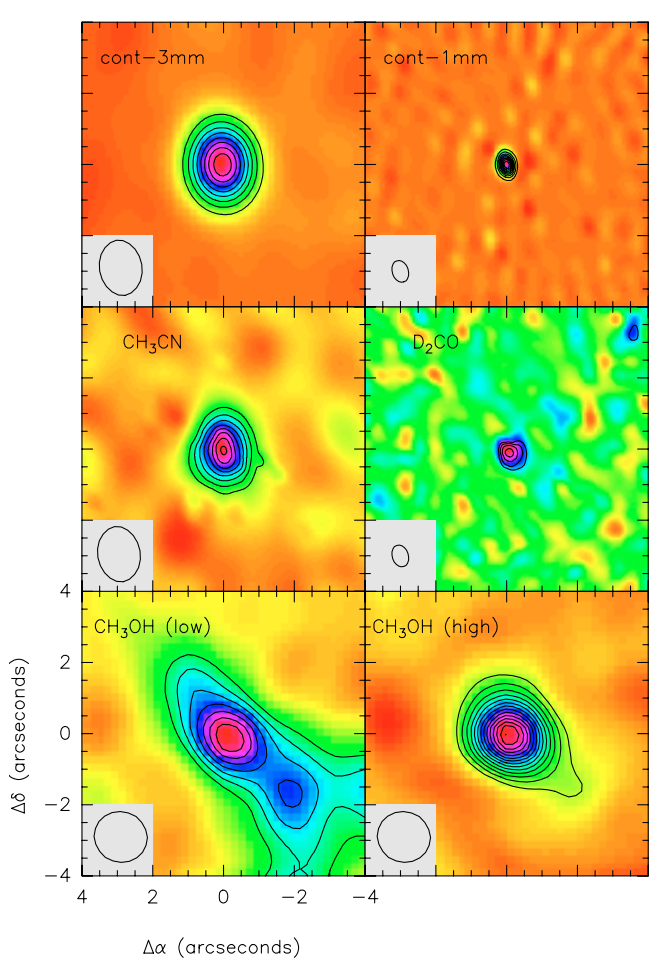

Fig. 1. Interferometric maps of the Class 0 protostar NGC 7129-FIRS 2 in the continuum at $1 \mathrm{~mm}$ and $3 \mathrm{~mm}$, and the following molecular lines: $\mathrm{CH}_{3} \mathrm{CN} 5 \rightarrow 4, \mathrm{D}_{2} \mathrm{CO} 4_{04} \rightarrow 3_{03}, \mathrm{CH}_{3} \mathrm{OH}$ $5_{1,4} \rightarrow 4_{1,4}$. The panel marked with " $\mathrm{CH}_{3} \mathrm{OH}$ (high)" shows the integrated intensity map of the lines $5_{3,1} \rightarrow 4_{3,1}, 5_{3,2} \rightarrow 4_{3,2}, 5_{2,2} \rightarrow 4_{2,2}$, and $5_{3,3} \rightarrow 4_{3,3}$. Contour levels are: a) 10 to 60 by $5 \mathrm{mJy} / \mathrm{beam}$; b) 20 to 160 by $20 \mathrm{mJy} /$ beam; c) 0.6 to 2.7 by $0.3 \mathrm{Jy} /$ beam $\mathrm{km} \mathrm{s}^{-1}$; d) 0.3 to 0.6 by $0.1 \mathrm{Jy} /$ beam $\mathrm{km} \mathrm{s}^{-1}$; e) 1 to 4 by $0.5 \mathrm{Jy} /$ beam $\mathrm{km} \mathrm{s}^{-1}$; f) 1 to 7.5 by $0.5 \mathrm{Jy} / \mathrm{beam} \mathrm{km} \mathrm{s}^{-1}$.

images show an intense and compact source located at $\operatorname{RA}(2000)=21: 43: 01.684, \operatorname{Dec}(2000)=66: 03: 23.619$, the position named MM1 in Paper I. There is no indication of the presence of the second and more extended source MM2, which is very likely resolved out in the high angular resolution image.

We modeled the visibilities for deeper insight into the source structure. In Fig. 2 we show the $92 \mathrm{GHz}$ and $230 \mathrm{GHz}$ visibilities vs. projected baselines in units of wavelength. The $92 \mathrm{GHz}$ visibilities scaled by a factor of 10.5 match the $230 \mathrm{GHz}$ visibilities perfectly. This suggests that both emissions are arising exactly in the same region. The spectral index is $\alpha=2.56$. That the $3 \mathrm{~mm}$ and $1.3 \mathrm{~mm}$ emissions arise in the same region and the spectral index is larger than 2 is consistent with dust thermal emission. We used the highangular resolution $1.3 \mathrm{~mm}$ image to model the continuum emission. Several models (elliptical Gaussian, disk, $F_{v}=v^{-2}$, $F_{v}=v^{-3}$, and an elliptical Gaussian+point source) were used to fit the visibilities. The best fit was obtained with the elliptical Gaussian+point source model. The $H P F W$ of the elliptical Gaussian is $\sim 650 \times 900 \mathrm{AU}$ and the flux $0.43 \mathrm{Jy}$. The point source has a flux of $0.13 \mathrm{Jy}$. The total flux of the compact $1.3 \mathrm{~mm}$ component is $\sim 0.56 \mathrm{Jy}$, which implies a total (dust + gas) mass of $2 M_{\odot}$ assuming a standard dust temperature of $100 \mathrm{~K}$ and a dust emissivity $k_{v}=0.015(1300 /$ $\lambda(\mu \mathrm{m})) \mathrm{cm}^{2} \mathrm{~g}^{-1}$. Considering the uncertainty in the value of the
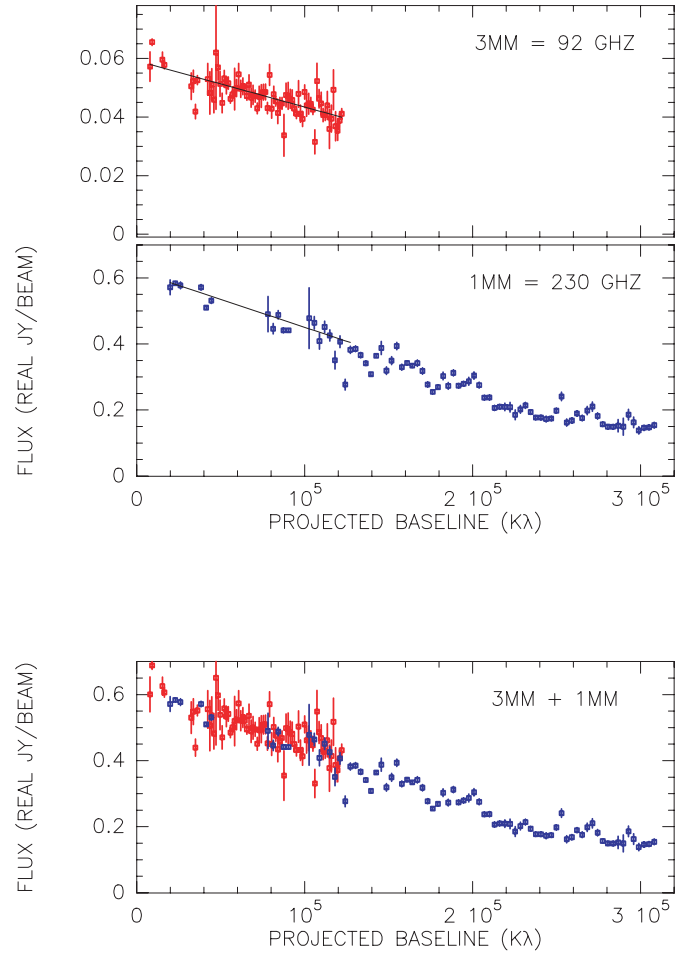

Fig. 2. Continuum visibilities at $92 \mathrm{GHz}$ and $230 \mathrm{GHz}$ vs. projected baselines in units of wavelength. In the bottom panel we show the $92 \mathrm{GHz}$ and the $230 \mathrm{GHz}$ visibilities together. The $92 \mathrm{GHz}$ visibilities were scaled by a factor of 10.5 to match the $230 \mathrm{GHz}$ ones. The perfect match between the $92 \mathrm{GHz}$ and $230 \mathrm{GHz}$ visibilities suggests that both emissions arise in the same region as expected in the case of dust thermal emission.

dust temperature, which could range between $50 \mathrm{~K}$ and $300 \mathrm{~K}$, this mass is accurate within a factor of 4 .

We speculate about the possibility that the point source is an accretion disk. In this case, the mass of the disk would be $\sim 0.3-0.8 M_{\odot}$. This value is 30 times higher than that found by Fuente et al. (2003) in the Herbig Be star R Mon, but it is similar to the one found by Rodríguez et al. (2005) in the Class 0 low-mass protostar IRAS 16293-2422B. Thus if our assumption is confirmed, the difference in the disk occurrence and masses between IM and low mass stars would be related to a short timescale for the disk dissipation instead of to differences in the first stages of the star formation. This interpretation is in line with Fuente et al. (2003).

\section{Molecular line images}

\section{1. $\mathrm{CH}_{3} \mathrm{CN}$}

The interferometric image of the Class 0 protostar NGC 7129FIRS 2 in the $\mathrm{CH}_{3} \mathrm{CN} 5 \rightarrow 4$ line shows a compact source at the same position and with the same size as the $1 \mathrm{~mm}$ continuum source (Fig. 1). The total agreement in position and size between the continuum and the $\mathrm{CH}_{3} \mathrm{CN}$ emissions points out to the existence of a well-differentiated compact component in the protostellar envelope. 
Table 2. Gaussian fits to the line and continuum emission.

\begin{tabular}{lcccc}
\hline \hline & \multicolumn{2}{c}{ Position $^{a}$} & $\begin{array}{c}\text { Integrated intensity }^{b} \\
(\mathrm{Jy})\end{array}$ & $\begin{array}{c}\text { Size }^{c} \\
(\operatorname{arcseconds})\end{array}$ \\
\hline Continuum 3 mm & $21: 43: 01.7$ & $66: 03: 23.6$ & $0.0565(0.0005)$ & $0.66(0.02) \times 0.54(0.02)$ \\
Continuum 1 mm & $21: 43: 01.7$ & $66: 03: 23.7$ & $0.4278(0.0047)$ & $0.72(0.01) \times 0.52(0.01)$ \\
& $21: 43: 01.7$ & $66: 03: 23.6$ & $0.1276(0.0046)$ & Point source $\left(<0.3^{\prime \prime}\right)$ \\
$\mathrm{CH}_{3} \mathrm{CN} 5_{k} \rightarrow 4_{k}$ & $21: 43: 01.7$ & $66: 03: 23.6$ & $0.0734(0.0060)$ & $0.63(0.22) \times 0.46(0.19)$ \\
$\mathrm{D}_{2} \mathrm{CO} 4_{04} \rightarrow 3_{0,3}$ & $21: 43: 01.7$ & $66: 03: 23.6$ & $0.76(0.6)$ & $0.58(0.24) \times 0.40(0.24)$ \\
\hline
\end{tabular}

${ }^{a}$ The absolute positional precision is $\leq 0.3^{\prime \prime}$.

${ }^{b}$ Systematic calibration errors are not included in the error budget associated to the integrated intensity.

${ }^{c}$ Deconvolved by the synthesized interferometric beam.
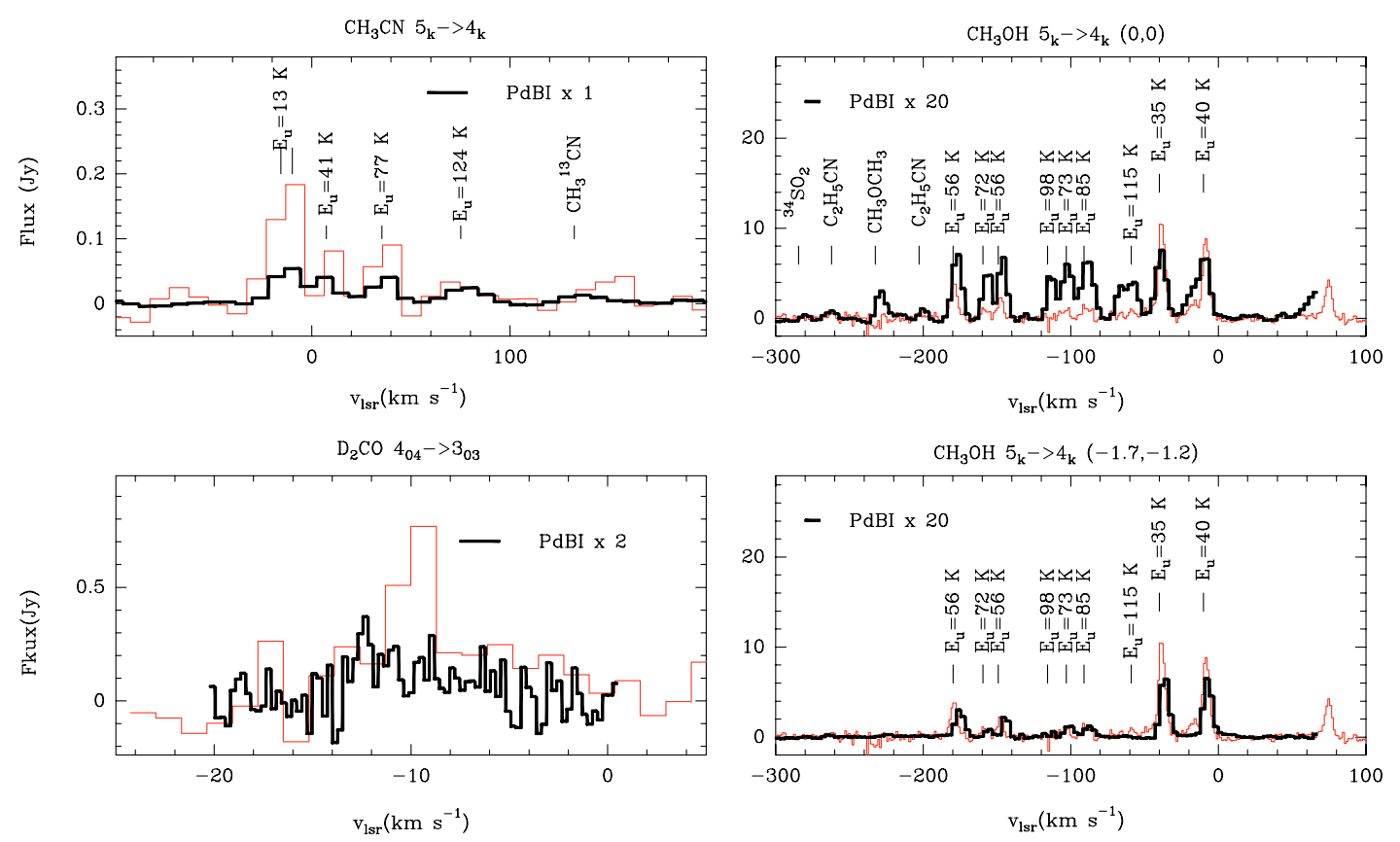

Fig. 3. Comparison between the single-dish (thin histograms) and interferometric (thick histograms) spectra towards the hot core position. The interferometric spectrum was scaled for an easier comparison. Note that we recovered all the flux for the $\mathrm{CH}_{3} \mathrm{CN}$ line with the highest upper state energy.

Because of the rotational structure of $\mathrm{CH}_{3} \mathrm{CN}$, one can observe several lines at different energies very close in frequency. In Fig. 3 we show the interferometric spectrum of the $\mathrm{CH}_{3} \mathrm{CN}$ $5 \rightarrow 4$ line compared to the single-dish spectrum reported in Paper II. The interferometer has recovered $100 \%$ of the flux of the highest upper state energy component $\left(E_{u}=124 \mathrm{~K}\right)$ (see Fig. 3). However, only $37 \%$ of the flux of the lowest energy component $\left(E_{u}=13 \mathrm{~K}\right)$ was recovered (see Fig. 3). This suggests that while the emission of the low energy $\mathrm{CH}_{3} \mathrm{CN}$ lines mainly arises in the cooler and more extended envelope, the emission of the high energy lines arises entirely in the hot core. In addition to the $\mathrm{CH}_{3} \mathrm{CN}$ K-ladder we also detected the $\mathrm{CH}_{3}{ }^{13} \mathrm{CN} 5_{0} \rightarrow 4_{0}$ line which allows us to estimate the opacity of the $\mathrm{CH}_{3} \mathrm{CN}$ line.

The $\mathrm{CH}_{3} \mathrm{CN}$ column density was estimated using the rotational diagram technique. The low energy lines are expected to be optically thick in the hot core. This is confirmed by our interferometric observations. In particular, we measure $I\left(\mathrm{CH}_{3} \mathrm{CN}\right.$ $\left.5_{0} \rightarrow 4_{0}\right) / I\left(\mathrm{CH}_{3}{ }^{13} \mathrm{CN}_{5} \rightarrow 4_{0}\right) \sim 2.4$, which implies an opacity $\sim 25$ for the main isotope line assuming ${ }^{12} \mathrm{C} /{ }^{13} \mathrm{C}=70$. We corrected by the opacity effect and derived $T_{\text {rot }} \sim 54 \mathrm{~K}$ and a beam averaged column density $N\left(\mathrm{CH}_{3} \mathrm{CN}\right) \sim 1.1 \times$ $10^{16} \mathrm{~cm}^{-2}$ for the hot core component using the $5_{0} \rightarrow 4_{0}$ and $5_{5} \rightarrow 4_{5}$ lines. The derived rotation temperature is in agreement, within the uncertainties, with the one derived from single dish data (Fuente et al. 2005). Since the low energy lines are optically thick in the hot core, we think that this agreement is fortuitous and due to the addition of two uncertainties which shift the estimated column density in opposite directions, the contribution of extended emission in the single-dish beam, and the opacity effect. From our interferometric observations, we obtained $X\left(\mathrm{CH}_{3} \mathrm{CN}\right) \sim 7.0 \times 10^{-9}$ in the $2 M_{\odot}$ hot core in FIRS 2 assuming a source size of $0.72^{\prime \prime} \times 0.52^{\prime \prime}$ (see Table 5). This fractional abundance is similar to that measured in massive hot cores and slightly larger than those derived in hot corinos.

For comparison, we estimated the $\mathrm{CH}_{3} \mathrm{CN}$ abundance in the cooler and extended envelope. For this aim, we subtracted the emission of the compact component from the single-dish spectrum and analyzed the result using a rotational diagram. The opacity effect is expected to be less important in the low 
density envelope than in the hot core. We derived $T_{\text {rot }}=12 \mathrm{~K}$ and a beam averaged column density of $5.8 \times 10^{11} \mathrm{~cm}^{-2}$ $\left(H P B W=25^{\prime \prime}\right)$. Using the $\mathrm{H}_{2}$ column density derived from single-dish continuum observations by Fuente et al. (2001), we estimated a $\mathrm{CH}_{3} \mathrm{CN}$ fractional abundance of $\sim 3 \times 10^{-11}$ in the extended component, i.e. about 3 orders of magnitude lower than in the compact component.

Summarizing, our $\mathrm{CH}_{3} \mathrm{CN}$ interferometric data show the existence of a compact $(900 \times 650 \mathrm{AU})$ source characterized by a high kinetic gas temperature $(>50 \mathrm{~K})$ and enhanced $\mathrm{CH}_{3} \mathrm{CN}$ abundance $\left(X\left(\mathrm{CH}_{3} \mathrm{CN}\right) \sim 7.0 \times 10^{-9}\right)$ in the Class $0 \mathrm{IM}$ protostar FIRS 2. This constitutes definite proof of the existence of a hot core in this IM protostar which, to our knowledge, is the first IM hot core detected so far.

\section{2. $\mathrm{CH}_{3} \mathrm{OH}$}

We observed the $\mathrm{CH}_{3} \mathrm{OH} J_{k, k^{\prime}}=5_{k, k^{\prime}} \rightarrow 4_{k, k^{\prime}}$ lines towards FIRS 2 using the $2.5 \mathrm{MHz}$ wide band correlator, which allowed us to observe most of the $\mathrm{CH}_{3} \mathrm{OH} J_{k, k^{\prime}}=5_{k, k^{\prime}} \rightarrow 4_{k, k^{\prime}}$ components simultaneously. In Fig. 1 we show the total integrated intensity map of the $\mathrm{CH}_{3} \mathrm{OH} 5_{1,4} \rightarrow 4_{1,4}$ line which is the one observed with the lowest upper stage energy $\left(E_{u}=35 \mathrm{~K}\right)$. The emission of this component presents two maxima; the first one is spatially coincident with the hot core while the second is located at the position $\left(-2^{\prime \prime},-1.5^{\prime \prime}\right)$. This position lies on the axis of the outflow FIRS 2-out 1. In Fig. 1 we also show the integrated intensity map of the $5_{3,1} \rightarrow 4_{3,1}, 5_{3,2} \rightarrow 4_{3,2}, 5_{2,2} \rightarrow 4_{2,2}$, and $5_{3,3} \rightarrow 4_{3,3}$ lines (panel indicated as $\mathrm{CH}_{3} \mathrm{OH}$ (high) in Fig. 1). These lines are partially overlapped in our spectrum and all of them have upper state energies $>50 \mathrm{~K}$. In this case, the emission mainly arises in the hot core with a very weak contribution of the second maximum. Thus, the morphology of the $\mathrm{CH}_{3} \mathrm{OH}$ emission shows the existence of two components, a compact one that is spatially coincident with the hot core (hereafter hot core component) and an extended one that is very likely associated with the outflow FIRS 2-out 1 (hereafter outflow component).

In Fig. 3 we compare the interferometric spectra for the hot core and outflow components with the single-dish spectrum at the central position. The two components present differences in their kinematics and excitation conditions. The emission of the $\mathrm{CH}_{3} \mathrm{OH}$ lines towards the hot core is centered at a velocity $-10 \pm 1 \mathrm{~km} \mathrm{~s}^{-1}$, while the emission of the outflow component is centered at $-6 \pm 1 \mathrm{~km} \mathrm{~s}^{-1}$. The ${ }^{12} \mathrm{CO} 2 \rightarrow 1$ and $\mathrm{SiO} 2 \rightarrow 1$ maps of the outflow FIRS 2-out 1 reported in Paper II show that this velocity, $-6 \pm 1 \mathrm{~km} \mathrm{~s}^{-1}$, is characteristic of the high velocity bullet $\mathrm{R} 1$, reinforcing our association of this emission with the bipolar outflow.

Different excitation conditions characterize the hot core and outflow components. This is clearly seen when one compares the relative intensities of the high and low energy lines in the methanol spectra towards the two studied positions. All the $\mathrm{CH}_{3} \mathrm{OH}$ lines have similar intensities towards the hot core. However, high energy lines are a factor $\sim 5$ weaker than the low energy ones towards the outflow position (see Fig. 3). We derived the rotation temperature and the beam averaged
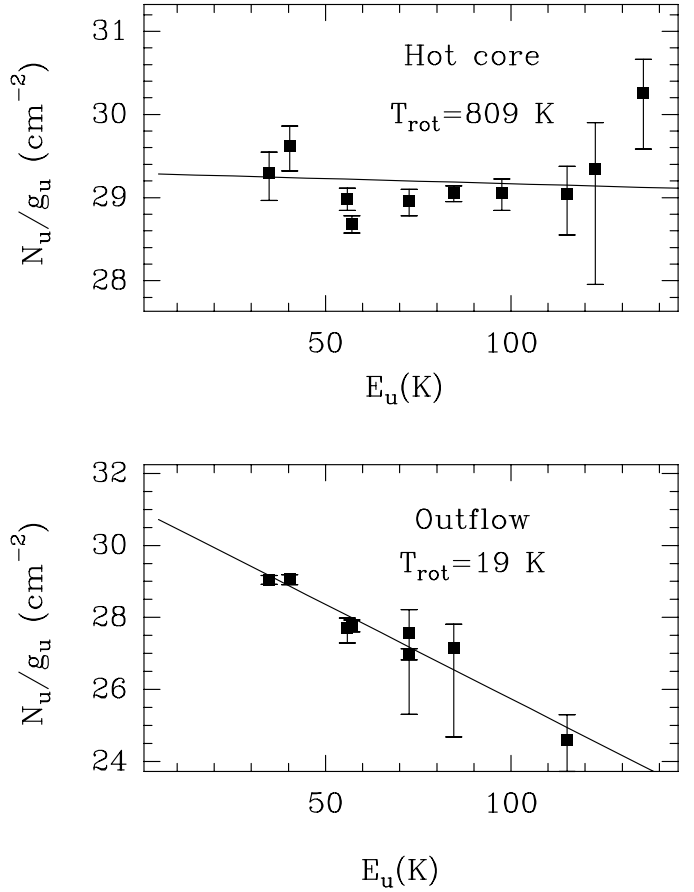

Fig. 4. Rotational diagram of $\mathrm{CH}_{3} \mathrm{OH}$ towards the hot core and the outflow condensation. Note that the rotation temperature towards the hot core is much higher than towards the outflow condensation.

$\mathrm{CH}_{3} \mathrm{OH}$ column density for the hot core using the rotational diagram technique (see Fig. 4 ), and obtained $T_{\text {rot }}=809 \mathrm{~K}$ and $N\left(\mathrm{CH}_{3} \mathrm{OH}\right)=1.5 \times 10^{17} \mathrm{~cm}^{-2}$. This temperature is quite high compared the one obtained from the $\mathrm{CH}_{3} \mathrm{CN}$ lines. Similar to the case of $\mathrm{CH}_{3} \mathrm{CN}$, the low energy lines are very likely optically thick, and the derived rotation temperature is an upper limit to the actual one. To have an estimate of the uncertainty due to the unknown rotation temperature in the $\mathrm{CH}_{3} \mathrm{OH}$ column density, we calculated the $\mathrm{CH}_{3} \mathrm{OH}$ column density from the flux of the $5_{4,3} \rightarrow 4_{4,3}$ line $\left(E_{u}=104 \mathrm{~K}\right)$ assuming $T_{\text {rot }}=50 \mathrm{~K}$. We obtain $N\left(\mathrm{CH}_{3} \mathrm{OH}\right)=4.9 \times 10^{16} \mathrm{~cm}^{-2}$. Thus, we consider that our beam averaged column density estimate is accurate within a factor of 3. Assuming $T_{\text {rot }}=50 \mathrm{~K}$, the derived $\mathrm{CH}_{3} \mathrm{OH}$ fractional abundance is $3.0 \times 10^{-8}$ in the hot core. This abundance is similar to that measured in the prototypical massive hot core OMC1.

For comparison, we estimated the rotation temperature and methanol column density for the outflow condensation (see Fig. 4), and obtained $T_{\text {rot }}=19 \mathrm{~K}$ and $N\left(\mathrm{CH}_{3} \mathrm{OH}\right)=2.9 \times$ $10^{15} \mathrm{~cm}^{-2}$. The low rotational temperature of this component supports our interpretation of it being associated with the outflow (postshocked material) instead of with the hot core. We did not detect a millimeter continuum counterpart for this condensation in our interferometric $1 \mathrm{~mm}$ map. In principle, this could be a sensitivity problem. From our interferometric image and assuming $T_{\text {dust }}=100 \mathrm{~K}$, we derive a $3 \times \sigma$ upper limit of $\sim 0.01 M_{\odot}$ for the mass of the condensation associated with the methanol clump. This would imply $X\left(\mathrm{CH}_{3} \mathrm{OH}\right)>3.8 \times 10^{-7}$ in this clump. We can alternatively think that this clump is not the result of a hydrogen density enhancement at this position. For example, it could be produced by a local enhancement of 
the $\mathrm{CH}_{3} \mathrm{OH}$ abundance because of the shocks associated with the bipolar outflow. To estimate the methanol abundance in this case, we calculated the $\mathrm{H}_{2}$ column density from the single-dish ${ }^{13} \mathrm{CO}$ observations reported in Paper II. At the velocities of the bullet $\mathrm{R} 1$, the ${ }^{13} \mathrm{CO}$ column density averaged in the $30 \mathrm{~m}$ beam is $\sim 3 \times 10^{15} \mathrm{~cm}^{-2}$. If we assume uniform distribution of the molecular gas in the beam, we find that the $\mathrm{CH}_{3} \mathrm{OH}$ abundance is similar to that of ${ }^{13} \mathrm{CO}$ for which we assume a standard value of $\sim 10^{-6}$. Obviously, this is an upper limit to the actual abundance since we expect some clumpiness. Thus, the $\mathrm{CH}_{3} \mathrm{OH}$ abundance in the outflow condensation could be larger than in the hot core and reach values close to $10^{-6}$. This high value of the methanol abundance is consistent with those found in the molecular bullets associated with low-mass bipolar outflows (see e.g. Bachiller \& Pérez-Gutierrez 1997).

In addition to the $\mathrm{CH}_{3} \mathrm{OH}$ lines, the spectrum towards the hot core show several lines that are weaker towards the outflow condensation. We identified these lines as belonging to the complex molecules $\mathrm{CH}_{3} \mathrm{OCH}_{3}$ and $\mathrm{C}_{2} \mathrm{H}_{5} \mathrm{CN}$. We also have a tentative detection of the sulphuretted species ${ }^{34} \mathrm{SO}_{2}$. The detection of complex saturated molecules corroborates the existence of a hot core in this source and reveals the rich and complex chemistry associated with it.

\section{3. $\mathrm{D}_{2} \mathrm{CO}$}

The interferometric map of $\mathrm{D}_{2} \mathrm{CO}$ shows a compact source located at the position of the hot core (see Fig. 1). The size of the $\mathrm{D}_{2} \mathrm{CO}$ emitting region is similar to that of $\mathrm{CH}_{3} \mathrm{CN}$. However, the linewidth of the $\mathrm{D}_{2} \mathrm{CO}$ line is $\sim 11 \mathrm{~km} \mathrm{~s}^{-1}$, larger than those of the $\mathrm{CH}_{3} \mathrm{CN}$ and $\mathrm{CH}_{3} \mathrm{OH}$ lines, which are typically $\sim 6-8 \mathrm{~km} \mathrm{~s}^{-1}$. Assuming the standard inside-outside collapse model, the largest linewidth of the $\mathrm{D}_{2} \mathrm{CO}$ line is consistent with the emission arising in an inner region of the protostellar envelope. In a simple calculation, an accretion velocity of $\sim 5 \mathrm{~km} \mathrm{~s}^{-1}$ corresponds to a radius of $\sim 130 \mathrm{AU}$ assuming a dynamical age of $\sim 10^{5} \mathrm{yr}$ and $\mathrm{d} M / \mathrm{d} t=5 \times 10^{-5} M_{\odot} \mathrm{yr}^{-1}$. A similar radius was found by Maret et al. (2004) for the region where the $\mathrm{H}_{2} \mathrm{CO}$ abundance is heavily enhanced because of evaporation of the grain mantles in low-mass protostars. This suggests a similar origin for the $\mathrm{D}_{2} \mathrm{CO}$ molecules in the hot core.

Our previous single-dish spectrum was too noisy to detect this wide emission (Paper II). Recently, we took a good signal-to-noise ratio spectrum to compare with the interferometric observations (see Fig. 3). The single-dish spectrum shows two components, a narrow one at the velocity of the ambient cloud (already detected in Paper II), which is completely missed in our interferometric observations, and a wide one with a linewidth similar to that of the PdB spectrum. Even in this wide component the interferometer recovers only $50 \%$ of the flux, suggesting that part of them arises in a more extended area.

A narrow and wide components with linewidths similar to those of $\mathrm{D}_{2} \mathrm{CO}$ were also detected in the single dish spectra of the $\mathrm{H}_{2} \mathrm{CO}$ lines (Paper II). In Paper II, we separately mapped the narrow and wide components. These maps revealed that both components are associated with the outflow. While the wide component arises in the jet, the emission of the narrow component was more intense in the interface between the jet and the molecular cloud suggesting that it is tracing the molecular gas being swept up by the jet. We propose that the missed flux in our interferometric $\mathrm{D}_{2} \mathrm{CO}$ image is probably arising in a more extended component associated with the bipolar outflow similar to the case of $\mathrm{H}_{2} \mathrm{CO}$.

Our interferometric image shows that, in addition to the extended component, there is an intense and compact $\mathrm{D}_{2} \mathrm{CO}$ component associated with the hot core. We estimated the $\mathrm{D}_{2} \mathrm{CO}$ abundance in this component assuming $T_{\text {rot }}=50 \mathrm{~K}$ and obtained $N\left(\mathrm{D}_{2} \mathrm{CO}\right)=3.5 \times 10^{14} \mathrm{~cm}^{-2}$ and $X\left(\mathrm{D}_{2} \mathrm{CO}\right) \sim 1.4 \times$ $10^{-10}$. This fractional abundance is a factor of $\sim 2$ higher than that derived in Paper II for the whole envelope.

\section{4. $N_{2} D^{+}$}

We did not detect the $\mathrm{N}_{2} \mathrm{D}^{+} 3 \rightarrow 2$ line in our interferometric image. The rms of the image is $50 \mathrm{mJy} /$ beam, which is about $\sim 2 \%$ of the flux measured with the $30 \mathrm{~m}$ telescope (Paper II). Thus, almost $100 \%$ of the $\mathrm{N}_{2} \mathrm{D}^{+}$emission was missed in the interferometric observations. This implies that the emission of $\mathrm{N}_{2} \mathrm{D}^{+}$presents quite a uniform distribution across the envelope and suggests that it arises mainly in the cooler extended envelope. This is also in agreement with our interpretation in Paper II based on kinematical arguments. The linewidth of the $\mathrm{N}_{2} \mathrm{D}^{+} 3 \rightarrow 2$ line, $\sim 1 \mathrm{~km} \mathrm{~s}^{-1}$, is at least a factor of 2-3 smaller than for lines arising in the warm component, and similar to those of the molecules which are thought to be good tracers of the cold envelope, such as $\mathrm{H}^{13} \mathrm{CO}^{+}$and $\mathrm{N}_{2} \mathrm{H}^{+}$.

\section{Chemical complexity of the hot core in NGC 7129-FIRS 2}

In Fig. 5 we show the observed wide band spectra in the upper side band (USB) and lower side band (LSB) of the $1 \mathrm{~mm}$ receiver. We detected a real forest of lines in the $1 \mathrm{~mm}$ band. Identifying these lines is not an easy task. Since we have moderate spectral resolution $(2.5 \mathrm{MHz})$, several candidates can be found for each line. On the other hand, in most cases we have two or more lines overlapped. We tried to identify most of them with the following procedure; in a first step we selected our best candidates for the lines and estimated the column density of the carriers assuming optically thin emission and local thermodynamic equilibrium (LTE). In a few cases, we were able to estimate the rotation temperature because we had at least two "isolated" lines (see Tables 3 and 4). In the other cases, we assumed $T_{\text {rot }}=50 \mathrm{~K}$. In a second step, we synthesized the expected spectrum assuming the estimated column densities. The velocity profile was assumed to be Gaussian with a half power full width of $7 \mathrm{~km} \mathrm{~s}^{-1}$ and centered at the ambient cloud velocity, $-10 \mathrm{~km} \mathrm{~s}^{-1}$. Finally, we compared it with the observed spectrum and readjusted the molecular column densities to improve the fit. Tables 3 and 4 list the lines detected in the USB and LSB spectra with the most likely line identification.

Obviously, this identification procedure entails some ambiguity. We classified the species according to the reliability of their identification in two groups that are differentiated in 

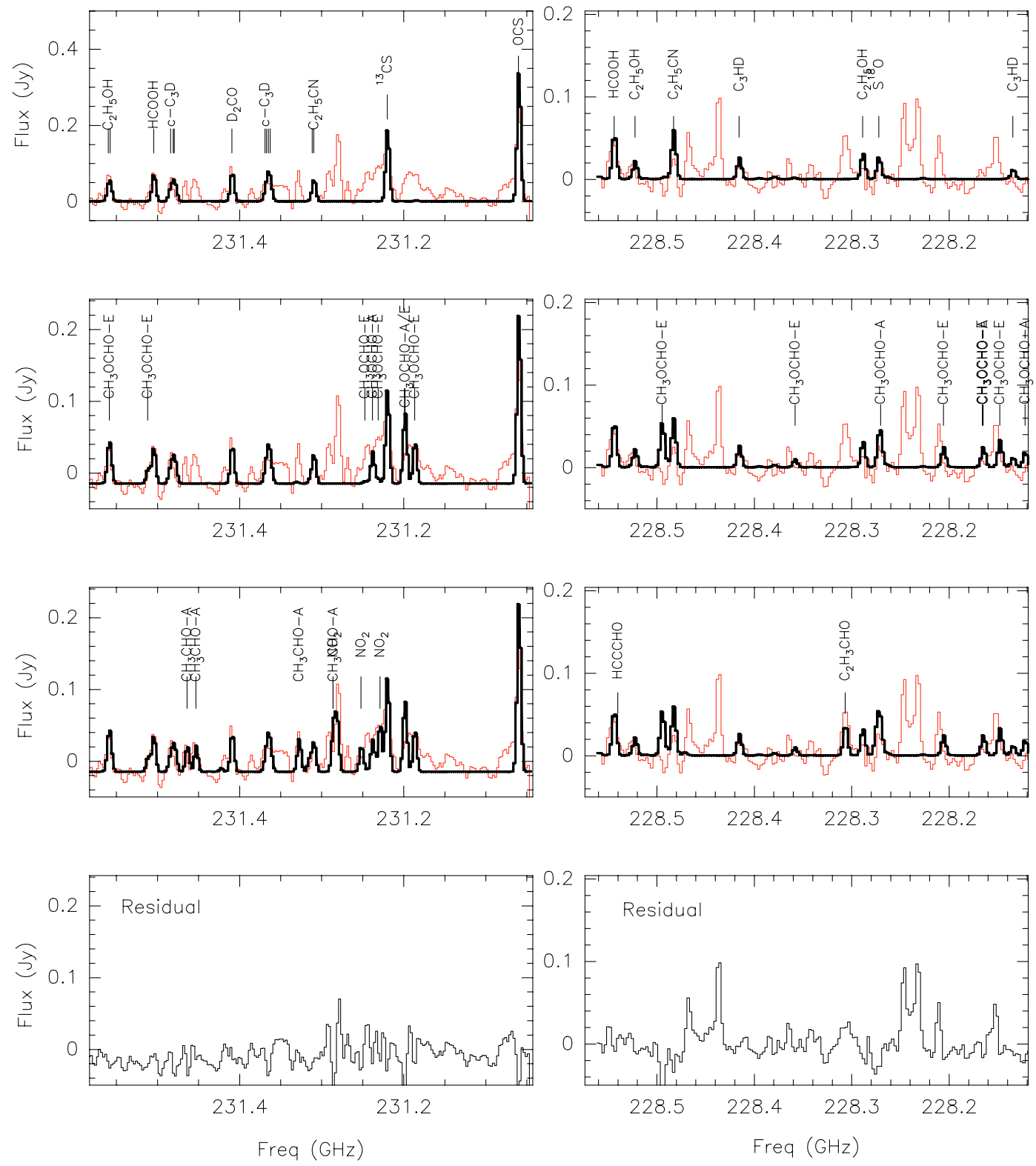

Fig. 5. Spectra of the upper side band (USB) and lower side band (LSB) of the $1 \mathrm{~mm}$ receiver obtained in our PdBI observations with our best guess for line identifications. For clarity, we divided the molecular species in three groups. The first group is formed by the more reliable identifications and $\mathrm{S}^{18} \mathrm{O}$. Beginning from the top, in the first line of panels we compare the observed spectra with the synthesized ones taking only this first group into account. In the second line, we add $\mathrm{CH}_{3} \mathrm{OCHO}-\mathrm{A} / \mathrm{E}$ and show the obtained synthesized spectra. Inclusion of these compounds improves agreement with the observational data. However, the large $\mathrm{CH}_{3} \mathrm{OCHO}-\mathrm{A} / \mathrm{E}$ abundance we derived from these observations raise some questions about this identification. In the third line of panels, we show the synthesized spectra after adding some other exotic compounds. The last line shows the residual spectra after subtracting our fit. Note that agreement between the synthesized and observed one is very good in the USB, but some lines remain unidentified in the LSB.

Table 5. The first group contains the most reliable identifications. In these cases, the observed frequencies are in total agreement with those calculated for these species. Moreover, the synthesized spectrum matches the observed one quite well (see top panel of Fig. 5). Finally, the derived fractional abundances are within the range of abundances measured for these species in hot cores. Within this group, we have OCS, ${ }^{13} \mathrm{CS}$, $\mathrm{C}_{2} \mathrm{H}_{5} \mathrm{CN}$, c- $\mathrm{C}_{3} \mathrm{D}, \mathrm{D}_{2} \mathrm{CO}, \mathrm{HCOOH}$, and $\mathrm{C}_{2} \mathrm{H}_{5} \mathrm{OH}$ in the USB and $\mathrm{C}_{2} \mathrm{H}_{5} \mathrm{OH}, \mathrm{C}_{3} \mathrm{HD}$ in the LSB. The lines of the first group species are indicated by bold characters in Tables 3 and 4 . The derived column densities and fractional abundances are shown in Table 5.

We put the complex molecules $\mathrm{CH}_{3} \mathrm{OCHO}-\mathrm{A}$ and $\mathrm{CH}_{3} \mathrm{OCHO}-\mathrm{E}$ (methyl formate) in the second group. Several lines of these species were detected in the USB and LSB (see second panels in Fig. 5). However, the derived fractional abundances in NGC 7129-FIRS 2 are unexpectedly high compared to those measured in hot corinos and massive hot cores (see 
Table 3. USB - $2.4 \mathrm{MHz}$.

\begin{tabular}{|c|c|c|c|c|}
\hline Freq. & Molecule & Transition & $\operatorname{Int}^{a}$ & $\overline{E_{u}(\mathrm{~K})}$ \\
\hline 231060.98 & OCS & $19 \rightarrow 18$ & -2.6263 & 100 \\
\hline 231187.69 & $\mathrm{CH}_{3} \mathrm{OCHO}-\mathrm{E}$ & $21_{9,13} \rightarrow 21_{8,14}$ & -4.8522 & 179 \\
\hline 231199.96 & $\mathrm{CH}_{3} \mathrm{OCHO}-\mathrm{A}$ & $21_{9,12} \rightarrow 21_{8,13}$ & -4.8484 & 179 \\
\hline 231200.14 & $\mathrm{CH}_{3} \mathrm{OCHO}-\mathrm{E}$ & $21_{9,12} \rightarrow 21_{8,13}$ & -4.8522 & 179 \\
\hline 231220.99 & ${ }^{13} \mathrm{CS}$ & $5 \rightarrow 4$ & -4.2103 & 51 \\
\hline 231229.96 & $\mathrm{NO}_{2}$ & $4_{1,3} \rightarrow 4_{0,4}$ & -4.8527 & 12 \\
\hline 231239.07 & $\mathrm{CH}_{3} \mathrm{OCHO}-\mathrm{A}$ & $21_{9,13} \rightarrow 21_{8,14}$ & -4.8482 & 179 \\
\hline 231232.06 & $\mathrm{CH}_{3} \mathrm{OCHO}-\mathrm{E}$ & $29_{6,24} \rightarrow 29_{5,25}$ & -5.0160 & 253 \\
\hline 231248.31 & $\mathrm{CH}_{3} \mathrm{OCHO}-\mathrm{E}$ & $29_{6,24} \rightarrow 29_{4,26}$ & -5.3013 & 253 \\
\hline 231252.98 & $\mathrm{NO}_{2}$ & $4_{1,3} \rightarrow 4_{0,4}$ & -5.0896 & 12 \\
\hline 231279.18 & $\mathrm{CH}_{3} \mathrm{CHO}-\mathrm{A}$ & $12_{6,7} \rightarrow 11_{6,6}$ & -2.9783 & 142 \\
\hline 231279.18 & $\mathrm{CH}_{3} \mathrm{CHO}-\mathrm{A}$ & $12_{6,6} \rightarrow 11_{6,5}$ & -2.9783 & 142 \\
\hline 231286.40 & $\mathrm{CH}_{3} \mathrm{OH}$ & $10_{2,2} \rightarrow 9_{3,2}$ & -4.0689 & 154 \\
\hline 231287.14 & $\mathrm{NO}_{2}$ & $4_{1,3} \rightarrow 4_{0,4}$ & -5.0875 & 12 \\
\hline 231310.42 & $\mathbf{C}_{2} \mathbf{H}_{5} \mathbf{C N}$ & $26_{1,25} \rightarrow 25_{1,24}$ & -2.6517 & 142 \\
\hline 231320.82 & $\mathrm{CH}_{3} \mathrm{CHO}-\mathrm{A}$ & $12_{5,8} \rightarrow 11_{5,7}$ & -2.9001 & 117 \\
\hline 231320.97 & $\mathrm{CH}_{3} \mathrm{CHO}-\mathrm{A}$ & $12_{5,7} \rightarrow 11_{5,6}$ & -2.9001 & 117 \\
\hline 231365.80 & c- $C_{3} D$ & $5_{3,3} \rightarrow 4_{3,2}$ & -3.1276 & 28 \\
\hline 231410.27 & $\mathbf{D}_{2} \mathbf{C O}$ & $4_{0,4} \rightarrow 4_{0,3}$ & -2.4242 & 17 \\
\hline 231446.49 & $\mathrm{CH}_{3} \mathrm{CHO}-\mathrm{A}$ & $12_{4,9} \rightarrow 11_{4,8}$ & -2.8386 & 97 \\
\hline 231457.25 & $\mathrm{CH}_{3} \mathrm{CHO}-\mathrm{A}$ & $12_{4,8} \rightarrow 11_{4,7}$ & -2.8386 & 97 \\
\hline 231485.11 & c- $\mathbf{C}_{3} \mathbf{D}$ & $5_{3,3} \rightarrow 4_{3,2}$ & -3.4082 & 28 \\
\hline 231505.70 & НСООН & $10_{1,9} \rightarrow 9_{1,8}$ & -3.1963 & 53 \\
\hline 231511.81 & $\mathrm{CH}_{3} \mathrm{OCHO}-\mathrm{E}$ & $27_{4,24} \rightarrow 27_{2,25}$ & -5.3849 & 211 \\
\hline 231513.06 & $\mathrm{CH}_{3} \mathrm{OCHO}-\mathrm{E}$ & $27_{4,24} \rightarrow 27_{3,25}$ & -5.1294 & 211 \\
\hline 231558.55 & $\mathbf{C}_{2} \mathbf{H}_{5} \mathbf{O H}$ & $21_{5,17} \rightarrow 21_{4,18}$ & -3.6252 & 215 \\
\hline 231559.62 & $\mathrm{CH}_{3} \mathrm{OCHO}-\mathrm{E}$ & $27_{5,23} \rightarrow 27_{2,25}$ & -5.1292 & 211 \\
\hline 231560.90 & $\mathrm{C}_{2} \mathrm{H}_{5} \mathrm{OH}$ & $20_{5,16} \rightarrow 20_{4,17}$ & -3.6250 & 197 \\
\hline
\end{tabular}

${ }^{a}$ Base 10 logarithm of the integrated intensity in units of $\mathrm{nm}^{2}-\mathrm{MHz}$ as appears tabulated in the JPL molecular line catalogue (Pickett et al. 1998).

Table 5). One possibility is that the rotation temperature is higher than was assumed. To estimate the uncertainty due to the poorly known rotation temperature, we repeated the column density calculations with $T_{\text {rot }}=300 \mathrm{~K}$. The derived abundance is an order of magnitude lower and then agrees with that derived in IRAS 16293. This high rotation temperature could be explained if this complex molecule arises in an inner and hotter region than $\mathrm{CH}_{3} \mathrm{CN}$. If methyl formate cannot be produced in the gas phase, as suggested by the laboratory results of Horn et al. (2004), our findings may imply that this species is formed on grain surfaces and that its binding energy is larger than that of $\mathrm{CH}_{3} \mathrm{CN}$, thus requiring larger dust temperatures to desorb.

The rarer isotope $\mathrm{S}^{18} \mathrm{O}$ has also been included in the group of uncertain identifications. We have only one line of this molecule, $\mathrm{S}^{18} \mathrm{O} 9_{8} \rightarrow 8_{8}$ at $228.272 \mathrm{GHz}$, and the derived $\mathrm{S}^{18} \mathrm{O}$ abundance is unexpectedly high. In this case we could have a misidentification. There is a $\mathrm{CH}_{3} \mathrm{OCHO}-\mathrm{A}$ line at $228.270 \mathrm{GHz}$, which could be an alternative identification of the tentative $\mathrm{S}^{18} \mathrm{O}$ line if the methyl formate abundance is unexpectedly high.

The most uncertain identifications are $\mathrm{C}_{2} \mathrm{H}_{3} \mathrm{CHO}$, $\mathrm{CH}_{3} \mathrm{CHO}-\mathrm{A}, \mathrm{HCCCHO}$, and $\mathrm{NO}_{2}$. The observed frequencies are in agreement with those of $\mathrm{C}_{2} \mathrm{H}_{3} \mathrm{CHO}, \mathrm{CH}_{3} \mathrm{CHO}-\mathrm{A}$, and $\mathrm{HCCCHO}$ lines if the emission arises in gas at a velocity
Table 4. LSB - $2.4 \mathrm{MHz}$.

\begin{tabular}{|c|c|c|c|c|}
\hline Freq. & Molecule & Transition & $\operatorname{Int}^{a}$ & $\overline{E_{u}(\mathrm{~K})}$ \\
\hline 228122.07 & $\mathrm{CH}_{3} \mathrm{OCHO}-\mathrm{A}$ & $5_{4,2} \rightarrow 4_{2,3}$ & -7.1993 & 8 \\
\hline 228134.42 & c- $\mathrm{C}_{3} \mathrm{HD}$ & $15_{7,8} \rightarrow 15_{7,9}$ & -3.9965 & 288 \\
\hline 228147.97 & $\mathrm{CH}_{3} \mathrm{OCHO}-\mathrm{E}$ & $18_{6,13} \rightarrow 18_{2,16}$ & -5.9576 & 114 \\
\hline 228165.01 & $\mathrm{CH}_{3} \mathrm{OCHO}-\mathrm{A}$ & $18_{6,13} \rightarrow 18_{4,14}$ & -5.9537 & 114 \\
\hline 228165.86 & $\mathrm{CH}_{3} \mathrm{OCHO}-\mathrm{E}$ & $44_{14,31} \rightarrow 43_{11,32}$ & -5.9853 & 673 \\
\hline 228270.42 & $\mathrm{CH}_{3} \mathrm{OCHO}-\mathrm{A}$ & $24_{9,16} \rightarrow 24_{8,17}$ & -4.8334 & 220 \\
\hline 228272.26 & $\mathrm{~S}^{18} \mathrm{O}$ & $9_{8} \rightarrow 8_{8}$ & -3.9573 & 82 \\
\hline 228288.69 & $\mathrm{C}_{2} \mathrm{H}_{5} \mathrm{OH}$ & $11_{11,0} \rightarrow 10_{2,8}$ & -4.0647 & 107 \\
\hline 228205.84 & $\mathrm{CH}_{3} \mathrm{OCHO}-\mathrm{E}$ & $24_{9,16} \rightarrow 24_{8,17}$ & -4.9577 & 220 \\
\hline 228232 & Unidentified & & & \\
\hline 228245 & Unidentified & & & \\
\hline 228278.96 & $\mathrm{HCCCHO}$ & $25_{1,25} \rightarrow 24_{1,24}$ & -2.8001 & 135 \\
\hline 228310.56 & $\mathrm{C}_{2} \mathrm{H}_{3} \mathrm{CHO}$ & $13_{2,12} \rightarrow 12_{1,11}$ & -4.6934 & 36 \\
\hline 228358.20 & $\mathrm{CH}_{3} \mathrm{OCHO}-\mathrm{E}$ & $24_{9,15} \rightarrow 24_{8,17}$ & -5.4380 & 220 \\
\hline 228415.69 & c- $\mathrm{C}_{3} \mathrm{HD}$ & $11_{8,4} \rightarrow 11_{6,5}$ & -4.5197 & 169 \\
\hline 228434 & Unidentified & & & \\
\hline 228467 & Unidentified & & & \\
\hline 228483.13 & $\mathbf{C}_{2} \mathbf{H}_{5} \mathbf{C N}$ & $25_{2,23} \rightarrow 24_{2,22}$ & -5.4674 & 158 \\
\hline 228494.88 & $\mathrm{CH}_{3} \mathrm{OCHO}-\mathrm{E}$ & $14_{4,10} \rightarrow 14_{1,13}$ & -6.29083 & 62 \\
\hline 228522.71 & $\mathrm{C}_{2} \mathrm{H}_{5} \mathrm{OH}$ & $7_{3,4} \rightarrow 7_{2,6}$ & -4.3308 & 84 \\
\hline 228544.17 & НСООН & $10_{2,8} \rightarrow 9_{2,7}$ & -3.2324 & 62 \\
\hline
\end{tabular}

${ }^{a}$ The same as in Table 3 .

$v_{\text {lsr }} \approx-5 \mathrm{~km} \mathrm{~s}^{-1}$. This velocity is coincident with that of the bullet R1 (see Paper I) but is shifted by $\sim 5 \mathrm{~km} \mathrm{~s}^{-1}$ from that of the molecular cloud. Similarly, the fit to the USB spectrum improves if we include a high excitation methanol line (see Table 3) emitting at the velocities -5 and $-20 \mathrm{~km} \mathrm{~s}^{-1}$. These velocities are within the range of velocities associated with this Class 0 protostar but are shifted from that of the ambient cloud. $\mathrm{NO}_{2}$ is not a common molecule in hot cores, and the three lines observed in the spectrum are overlapped with others of more common hot core species. We have kept it in the table because its inclusion improves the fit to the observed spectrum.

In Fig. 5 we show the synthesized USB and LSB spectra assuming the molecular column densities listed in Table 3. The fit is quite good for the USB. However, it is poor for the LSB, where we still have 4 unidentified lines. This is quite frustrating taking into account that they are the most intense lines in this subband. The frequencies of the unidentified lines are $228467( \pm 5), 228434( \pm 5), 228245( \pm 5)$, and $228232( \pm 5) \mathrm{MHz}$, and we do not have any good candidates for them. There is a line of vibrationally excited $\mathrm{DC}_{3} \mathrm{~N}$ at the frequency of $228467.44 \mathrm{GHz}$. Emission of vibrationally excited $\mathrm{HC}_{3} \mathrm{~N}$ has been detected in massive hot cores (see e.g. Wyrowski et al. 1999). However, if this identification were correct, we would expect to see a vibrationally excited $\mathrm{HC}_{3} \mathrm{~N}$ line at least ten times more intense at a frequency of $228303 \mathrm{MHz}$, which was not detected. For the line at $228434 \mathrm{MHz}$ our best candidate is glycoaldehyde $\left(\mathrm{CH}_{2}(\mathrm{OH}) \mathrm{CHO}\right)$, which has already been observed in the interstellar medium (Hollis et al. 2004). However, the upper state energy of the line at 228434 $\mathrm{MHz}$ is too high $(>1400 \mathrm{~K})$ to be detectable in this source. The line at $228245 \mathrm{MHz}$ could be propadienone $\left(\mathrm{CH}_{2} \mathrm{CCO}\right)$, but in this case we should have detected other lines of this compound in the same spectrum. Finally, our best candidate for the line at 
Table 5. Fractional Abundances in the hot core of NGC 7129-FIRS 2 and comparison with other hot cores.

\begin{tabular}{|c|c|c|c|c|c|c|c|}
\hline Molecule & $T_{\text {rot }}(\mathrm{K})$ & $\begin{aligned} \mathrm{GC} 7129-\mathrm{FI} \\
\\
\sim 500 L_{\odot} \\
N^{*}\left(\mathrm{~cm}^{-2}\right)\end{aligned}$ & $\overline{\mathrm{S} 2}$ & $\begin{array}{c}\text { NGC } 1333 \text { IRAS4 A } \\
\sim 14 L_{\odot} \\
X^{b}\end{array}$ & $\begin{array}{c}\text { IRAS } 16293 \\
\sim 23 L_{\odot} \\
X^{c}\end{array}$ & $\begin{array}{c}\text { OMC 1 } \\
\sim 1.0 \times 10^{4} L_{\odot} \\
X^{d}\end{array}$ & $\begin{array}{c}\mathrm{G} 327.3-0.6 \\
\sim 10^{5} L_{\odot} \\
X^{e}\end{array}$ \\
\hline $\mathrm{H}_{2}$ & & $7.9 \times 10^{24}$ & 1 & 1 & 1 & 1 & 1 \\
\hline $\mathrm{CH}_{3} \mathrm{CN}$ & 54 & $5.5 \times 10^{16}$ & $7.0 \times 10^{-9}$ & $1.6 \times 10^{-9}$ & $1.0 \times 10^{-8}$ & $4 \times 10^{-9}$ & $7 \times 10^{-7}$ \\
\hline $\mathrm{CH}_{3} \mathrm{OH}$ & $50^{a}$ & $2.4 \times 10^{17}$ & $3.0 \times 10^{-8}$ & $<710^{-9}$ & $3.0 \times 10^{-7}$ & $1 \times 10^{-7}$ & $2 \times 10^{-5}$ \\
\hline $\mathrm{H}_{2} \mathrm{CO}$ & $50^{a}$ & $2.5 \times 10^{15}$ & $3.1 \times 10^{-10}$ & $2.0 \times 10^{-8}$ & $6.0 \times 10^{-8}$ & $7.0 \times 10^{-9}$ & \\
\hline $\mathrm{D}_{2} \mathrm{CO}$ & $50^{a}$ & $3.5 \times 10^{14}$ & $4.4 \times 10^{-11}$ & & $3.0 \times 10^{-9 g}$ & & \\
\hline${ }^{13} \mathrm{CS}$ & $50^{a}$ & $3.4 \times 10^{14}$ & $4.3 \times 10^{-11}$ & & & & \\
\hline OCS & $50^{a}$ & $2.4 \times 10^{16}$ & $3.0 \times 10^{-9}$ & & $1.0 \times 10^{-6}$ & $1 \times 10^{-8}$ & $>2 \times 10^{-9 h}$ \\
\hline $\mathrm{HCOOH}$ & 48 & $3.5 \times 10^{15}$ & $4.4 \times 10^{-10}$ & $4.6 \times 10^{-9}$ & $6.2 \times 10^{-8}$ & $8 \times 10^{-10}$ & $3 \times 10^{-10 h}$ \\
\hline $\mathrm{C}_{2} \mathrm{H}_{5} \mathrm{OH}$ & 75 & $2.0 \times 10^{16}$ & $2.5 \times 10^{-9}$ & & & $7 \times 10^{-10}$ & $3 \times 10^{-9 h}$ \\
\hline $\mathrm{C}_{2} \mathrm{H}_{5} \mathrm{CN}$ & $50^{a}$ & $3.2 \times 10^{15}$ & $4.0 \times 10^{-10}$ & $<1.2 \times 10^{-9}$ & $1.2 \times 10^{-8}$ & $3.0 \times 10^{-9}$ & $4 \times 10^{-7}$ \\
\hline$c-C_{3} D$ & $50^{a}$ & $2.5 \times 10^{15}$ & $3.1 \times 10^{-10}$ & & & & \\
\hline $\mathrm{c}-\mathrm{C}_{3} \mathrm{HD}$ & 50 & $1.8 \times 10^{17}$ & $2.3 \times 10^{-8}$ & & & & \\
\hline \multicolumn{8}{|c|}{ Uncertain identifications } \\
\hline $\mathrm{S}^{18} \mathrm{O}$ & $50^{a}$ & $2.6 \times 10^{16}$ & $3.3 \times 10^{-9}$ & & $2.6 \times 10^{-9}$ & $3 \times 10^{-10}$ & $>6 \times 10^{-13}$ \\
\hline $\mathrm{CH}_{3} \mathrm{OCHO}-\mathrm{E}$ & 36 & $4.0 \times 10^{18}$ & $5.0 \times 10^{-7}$ & $3.6 \times 10^{-8}$ & $2.3 \times 10^{-7}$ & $1 \times 10^{-8}$ & $2 \times 10^{-6}$ \\
\hline $\mathrm{CH}_{3} \mathrm{OCHO}-\mathrm{A}$ & $36^{a}$ & $3.3 \times 10^{18}$ & $4.1 \times 10^{-7}$ & $3.4 \times 10^{-8}$ & $1.7 \times 10^{-7}$ & $1 \times 10^{-8}$ & $2 \times 10^{-6}$ \\
\hline $\mathrm{CH}_{3} \mathrm{CHO}-\mathrm{A}$ & $50^{a}$ & $2.3 \times 10^{15}$ & $2.9 \times 10^{-10}$ & & $1.9 \times 10^{-8}$ & & $5 \times 10^{-10 h}$ \\
\hline HCCCHO & $50^{a}$ & $2.0 \times 10^{15}$ & $2.5 \times 10^{-10}$ & & & & \\
\hline $\mathrm{C}_{2} \mathrm{H}_{3} \mathrm{CHO}$ & $50^{a}$ & $5.0 \times 10^{16}$ & $6.3 \times 10^{-9}$ & & & & \\
\hline $\mathrm{CH}_{3} \mathrm{OCH}_{3}$ & $50^{a}$ & $1.9 \times 10^{15}$ & $2.4 \times 10^{-10}$ & $<2.8 \times 10^{-8}$ & $2.4 \times 10^{-7}$ & $8 \times 10^{-9}$ & $3 \times 10^{-8 h}$ \\
\hline${ }^{34} \mathrm{SO}_{2}$ & $50^{a}$ & $3.8 \times 10^{13}$ & $4.8 \times 10^{-12}$ & & $2.4 \times 10^{-8}$ & & \\
\hline $\mathrm{NO}_{2}$ & $50^{a}$ & $1.0 \times 10^{17}$ & $1.2 \times 10^{-8}$ & & & & \\
\hline
\end{tabular}

* Values derived for the NGC 7129-FIRS 2 hot core assuming a size of $\sim 0.72^{\prime \prime} \times 0.52^{\prime \prime}$. To derive the $\mathrm{H}_{2}$ column density we considered that the hot core mass $\left(2 M_{\odot}\right)$ is uniformly distributed in an area $\sim a b$ with $a=0.004 \mathrm{pc}\left(0.72^{\prime \prime}\right)$ and $b=0.003 \mathrm{pc}\left(0.52^{\prime \prime}\right)$.

${ }^{a}$ Assumed temperature.

${ }^{b}$ Values from Bottinelli et al. (2004) for a $0.0016 \mathrm{pc}\left(0.5^{\prime \prime}\right)$ source.

${ }^{c}$ Values from Cazaux et al. (2003) and Wakelam et al. (2004) for a $0.0016 \mathrm{pc}\left(2^{\prime \prime}\right)$ source.

${ }^{d}$ Values from Sutton et al. (1995) for a $0.034 \mathrm{pc}\left(14^{\prime \prime}\right)$ source.

${ }^{e}$ Values from Gibb et al. (2000) for a $0.03 \mathrm{pc}\left(2^{\prime \prime}\right)$ source.

${ }^{f}$ From Schoier et al. (2002).

${ }^{g}$ From Ceccarelli et al. (2001).

${ }^{h}$ From Gibb et al. (2000) for a 0.32 pc $\left(20^{\prime \prime}\right)$ source.

$228232 \mathrm{MHz}$ is cyclopropenone $\left(\mathrm{C}_{3} \mathrm{H}_{2} \mathrm{O}\right)$, and this molecule has never been detected in space. Since we have only one observable line in our spectra, we cannot confirm this detection.

In addition to the lines detected in the wide-band spectrum shown in Fig. 5, in Table 5 we also include the column densities derived for $\mathrm{CH}_{3} \mathrm{OCH}_{3}$ and ${ }^{34} \mathrm{SO}_{2}$, the species detected in the $\mathrm{CH}_{3} \mathrm{OH}$ spectrum shown in Fig. 3. Since we have only one line for these species, the derived column density is also uncertain.

\section{Discussion}

In Table 5 we list the derived abundances of the molecules detected in the hot core towards FIRS 2. A first look at the table reveals that FIRS 2 is rich in deuterated species $\left(\mathrm{D}_{2} \mathrm{CO}, \mathrm{c}-\mathrm{C}_{3} \mathrm{D}\right.$ and $\left.\mathrm{c}-\mathrm{C}_{3} \mathrm{HD}\right)$, sulphuretted compounds $\left({ }^{13} \mathrm{CS}\right.$, OCS), and complex $\mathrm{O}-$ and $\mathrm{N}$ - molecules $\left(\mathrm{HCOOH}, \mathrm{C}_{2} \mathrm{H}_{5} \mathrm{OH}, \mathrm{C}_{2} \mathrm{H}_{5} \mathrm{CN}\right)$. Taking the large uncertainty in the estimated fractional abundances into account, we centered our discussion on the most reliable identifications, i.e., the first group in Table 5.

\subsection{Deuterated species}

The deuterium fractionation of molecular species is a wellknown process in dense interstellar clouds (Watson 1974). In the gas phase, this is mainly driven by enhancement of the $\mathrm{H}_{2} \mathrm{D}^{+} / \mathrm{H}_{3}^{+}$ratio due to the exothermic proton-deuteron exchange reaction $\mathrm{H}_{3}^{+}+\mathrm{HD} \rightarrow \mathrm{H}_{2} \mathrm{D}^{+}+\mathrm{H}_{2}(+230 \mathrm{~K})$, which does not proceed backwards if the gas temperature is less than about $30 \mathrm{~K}$. This process is responsible for the deuteration of species such as $\mathrm{HCO}^{+}$and $\mathrm{N}_{2} \mathrm{H}^{+}$, via the reaction of $\mathrm{H}_{2} \mathrm{D}^{+}$with the parent species $\mathrm{CO}$ and $\mathrm{N}_{2}$, respectively, as observed in dense cloud cores (Butner et al. 1995; Willacy et al. 1998).

Another phenomenon which boosts the deuterium fractionation is the freeze-out of neutral species, in particular the abundant $\mathrm{O}$ and $\mathrm{CO}$, which are efficient destruction partners of $\mathrm{H}_{3}^{+}$ and $\mathrm{H}_{2} \mathrm{D}^{+}$in the gas phase. Their freeze out, known to happen in dense cold clouds (Williams et al. 1998; Caselli et al. 1999; Kramer et al. 1999; Bergin et al. 2002; Tafalla et al. 2002), further enhances the $\mathrm{H}_{2} \mathrm{D}^{+} / \mathrm{H}_{3}^{+}$abundance ratio (because of the enhanced formation rate of $\mathrm{H}_{2} \mathrm{D}^{+}$, and the reduced destruction rates for $\mathrm{H}_{2} \mathrm{D}^{+}$and $\mathrm{H}_{3}^{+}$) and the consequent transfer 


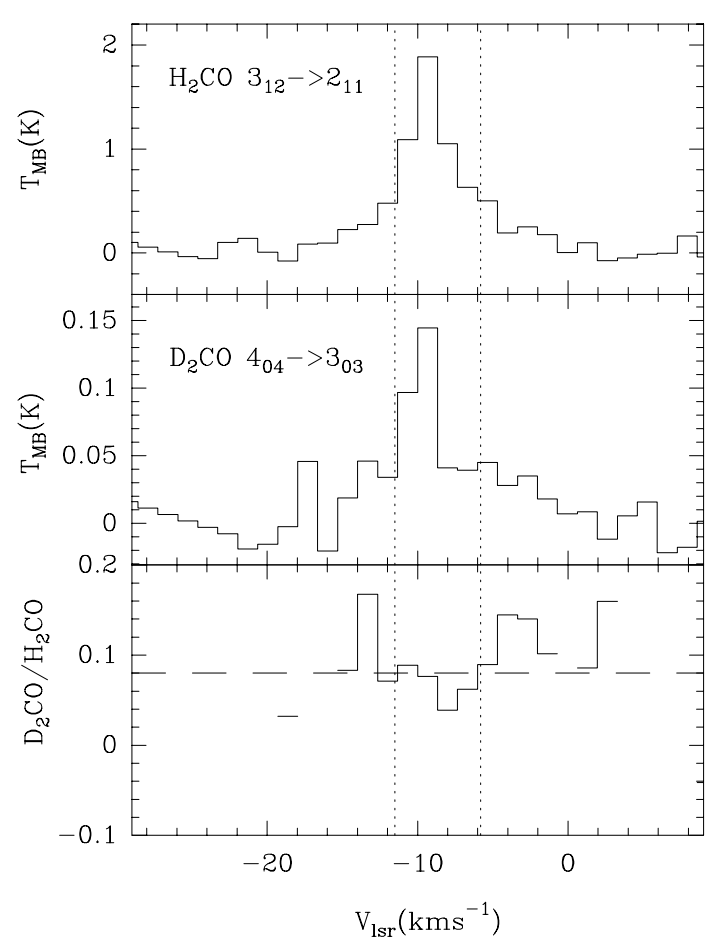

Fig. 6. Single-dish spectra of the $\mathrm{H}_{2} \mathrm{CO} \quad 3_{12} \rightarrow 2_{11}$ and $\mathrm{D}_{2} \mathrm{CO} 4_{04} \rightarrow 3_{03}$ lines towards FIRS 2. In the bottom panel we show the $\mathrm{I}\left(\mathrm{D}_{2} \mathrm{CO} 4_{04} \rightarrow 3_{03}\right) / \mathrm{I}\left(\mathrm{H}_{2} \mathrm{CO} 3_{12} \rightarrow 2_{11}\right)$ line ratio. Note that this ratio presents a minimum around the velocity of the ambient cloud $\left(\sim-10 \mathrm{~km} \mathrm{~s}^{-1}\right)$.

of the deuteron to gas-phase species (Caselli et al. 2002a; Bacmann et al. 2003; Crapsi et al. 2005). Once CO is highly depleted, multiple deuterated forms of $\mathrm{H}_{3}^{+}$can easily be produced (Roberts et al. 2003; Walmsley et al. 2004). Indeed, large abundances of $\mathrm{H}_{2} \mathrm{D}^{+}$and $\mathrm{D}_{2} \mathrm{H}^{+}$have been measured toward two pre-stellar cores (Caselli et al. 2003; Vastel et al. 2004). This has the consequence of enhancing the gaseous $\mathrm{D} / \mathrm{H}$ ratio to values around 0.1 , four orders of magnitude larger than the cosmic D abundance, which allows an efficient deuteration on grain surfaces (Tielens 1983; Charnley et al. 1997; Stantcheva $\&$ Herbst 2003).

We detected the deuterated species $\mathrm{D}_{2} \mathrm{CO}, \mathrm{c}-\mathrm{C}_{3} \mathrm{D}$, and c- $\mathrm{C}_{3} \mathrm{HD}$ towards FIRS 2. Moreover, the $\mathrm{D}_{2} \mathrm{CO}$ interferometric image shows that the $\mathrm{D}_{2} \mathrm{CO}$ emission is strongly peaked towards the hot core position. Recent theoretical and observational research suggests that the abundance of the nondeuterated species $\mathrm{H}_{2} \mathrm{CO}$ is enhanced by more than 2 orders of magnitude in the inner region of the envelope, where the dust temperature is higher than $100 \mathrm{~K}$ (Maret et al. 2004). The size of this region $(\sim 100 \mathrm{AU})$ is similar to that derived for the $\mathrm{D}_{2} \mathrm{CO}$ emission from the linewidth of the $\mathrm{D}_{2} \mathrm{CO}$ line. Assuming that evaporation is also the origin of $\mathrm{D}_{2} \mathrm{CO}$, the $\left[\mathrm{D}_{2} \mathrm{CO}\right] /\left[\mathrm{H}_{2} \mathrm{CO}\right]$ would tell us about the gaseous $\mathrm{D} / \mathrm{H}$ ratio during the pre-stellar phase of the parent clump. Unfortunately, we have no interferometric observations of $\mathrm{H}_{2} \mathrm{CO}$ and cannot derive an accurate $\left[\mathrm{D}_{2} \mathrm{CO}\right] /\left[\mathrm{H}_{2} \mathrm{CO}\right]$ ratio in the hot core. Trying to get an estimate of the deuteration degree of formaldehyde, we compared the single dish spectra of the $\mathrm{D}_{2} \mathrm{CO} 4_{04} \rightarrow 3_{03}$ and $\mathrm{H}_{2} \mathrm{CO} 3_{12} \rightarrow 2_{11}$ lines (see Fig. 6). Both lines have similar excitation conditions and frequency. The line intensity ratio in the narrow component is lower by a factor of 2 than in the wide component suggesting a higher $\left[\mathrm{D}_{2} \mathrm{CO}\right] /\left[\mathrm{H}_{2} \mathrm{CO}\right]$ ratio in the hot core. Assuming the same physical conditions for $\mathrm{D}_{2} \mathrm{CO}$ and $\mathrm{H}_{2} \mathrm{CO}$, we derived $\left[\mathrm{D}_{2} \mathrm{CO}\right] /\left[\mathrm{H}_{2} \mathrm{CO}\right] \sim 0.14 \pm 0.03$ in the hot core. This value is similar to the one found in pre-stellar cores and low-mass protostars (Caselli et al. 2002a; Bacmann et al. 2003; Ceccarelli et al. 2001; Loinard et al. 2003), and it implies large D/H ratios during the cold pre-stellar phase $(\mathrm{D} / \mathrm{H} \sim 0.5$, see Caselli et al. 2002ab; Stantcheva \& Herbst 2003).

In contrast to $\mathrm{D}_{2} \mathrm{CO}$, we have not detected $\mathrm{N}_{2} \mathrm{D}^{+}$in our interferometric observations. It is interesting to note that, whereas the deuteration of $\mathrm{N}_{2} \mathrm{H}^{+}$is a gas-phase process (which proceeds from the reaction between $\mathrm{N}_{2}$ and $\mathrm{H}_{2} \mathrm{D}^{+}$), the formation of $\mathrm{D}_{2} \mathrm{CO}$ seems to require surface chemistry (Ceccarelli et al. 2002). Therefore, our result is not surprising. In fact, as stated above, the deuteration in the gas phase is efficient only if the gas temperature is lower than, say, $30 \mathrm{~K}$. Thus, in a hot core, we do not expect to see $\mathrm{N}_{2} \mathrm{D}^{+}$. Moreover, in hot cores, where icy grain mantles evaporates, $\mathrm{CO}$ and $\mathrm{H}_{2} \mathrm{O}$ molecules are abundant in the gas phase, and they efficiently destroy $\mathrm{N}_{2} \mathrm{H}^{+}$(and $\mathrm{N}_{2} \mathrm{D}^{+}$), so that one does not expect to see large abundances of $\mathrm{N}_{2} \mathrm{H}^{+}$either. The non-detection of $\mathrm{N}_{2} \mathrm{D}^{+}$in the hot core associated with FIRS 2 confirms the interpretation given in Paper II that $\mathrm{N}_{2} \mathrm{D}^{+}$arises mainly in the cool envelope, and constitutes indirect proof of this chemical scheme. On the other hand, the large $\mathrm{D}_{2} \mathrm{CO} / \mathrm{H}_{2} \mathrm{CO}$ abundance ratio observed in the same position can be easily explained if the deuterium fractionation of formaldehyde happened on grain surfaces during the cold prestellar phase, and if icy mantles have recently evaporated, so that gas phase chemistry did not have enough time to reduce the abundance of the deuterated "parent" species.

\subsection{Sulphuretted species}

The abundances of sulphuretted molecules are very dependent on the stellar age. We compared the observed [CS]/[OCS] ratio in FIRS $2\left(\sim 1.1\right.$ assuming $\left.\mathrm{CS} /{ }^{13} \mathrm{CS}=89\right)$ with the Viti et al. (2004) model for a $5 M_{\odot}$ star. The measured ratio is consistent with the FIRS 2 being an evolved hot core with an age of $>1.0 \times 10^{5} \mathrm{yr}$. The detection of large species like $\mathrm{C}_{2} \mathrm{H}_{5} \mathrm{OH}$ also suggests that we are dealing with an evolved hot core, since these species are only formed at late times. Nevertheless, we would like to point out that determination of the hot core age using the sulphuretted molecules is quite uncertain since other factors like the ice mantle composition and the hot core kinetic temperature can also strongly affect the abundances of these species (Wakelam et al. 2004).

\subsection{Complex $\mathrm{O}$ - and $\mathrm{N}$-bearing molecules}

Theoretical models predict that the relative abundance of the $\mathrm{N}$-bearing and O-bearing complex molecules is dependent on the hot core temperature (Caselli et al. 1993; Rodgers \& Charnley 2001). The luminosity of FIRS 2 is a factor $>10$ larger than those of the two low mass hot corinos detected so far and a factor 200 lower than that of the massive protostar creating 
the Orion hot core. The intermediate kinetic temperature of the FIRS 2 hot core is expected to produce a differentiated chemistry of complex molecules. In order to better show the dependence of the complex molecule abundances on the protostellar luminosity, in Table 5 we compare the observed abundances towards FIRS 2 with those measured in prototypical hot corinos and massive hot cores. The luminosity of the objects listed in Table 5 varies by 4 orders of magnitude, thus one expects to detect some chemical differences between these hot cores.

However, comparing abundances between different hot cores (corinos) is difficult. First of all, since massive stars are usually located farther from the Sun than their low- and intermediate-mass counterparts, the abundances are derived on different spatial scales. The fractional abundances shown in Table 5 are averaged values in regions with sizes ranging from $\sim 0.002 \mathrm{pc}$ in NGC1333-IRAS 4A to $0.32 \mathrm{pc}$ in OMC1 and G327. These sizes are given by the angular resolution of the current instrumentation at the hot core distance. Thus, the derived abundances in massive hot cores would be severely underestimated in the case of sizes similar to those of hot corinos. On the other hand, we can have several hot cores in the studied region, especially in massive star-forming regions where clustering is more important and are located at greater distances. In order to minimize these problems, we normalized the studied abundances to those of the parent species.

As a first step, we normalized all the molecular abundances to that of $\mathrm{H}_{2} \mathrm{CO}$ and $\mathrm{CH}_{3} \mathrm{OH}$. These species are thought to be the "parent" molecules of complex O-bearing species, although there is some doubt in the particular case of methyl formate (Horn et al. 2004). In Fig. 7a, we show the relative abundances of $\mathrm{HCOOH}, \mathrm{CH}_{3} \mathrm{OH}, \mathrm{CH}_{3} \mathrm{CN}$, and $\mathrm{C}_{2} \mathrm{H}_{5} \mathrm{CN}$ with respect to $\mathrm{H}_{2} \mathrm{CO}$ as a function of the luminosity. Since we have no interferometric observations of $\mathrm{H}_{2} \mathrm{CO}$ in FIRS 2, we estimated the $\mathrm{H}_{2} \mathrm{CO}$ abundance assuming $\left[\mathrm{D}_{2} \mathrm{CO}\right] /\left[\mathrm{H}_{2} \mathrm{CO}\right]=0.014$. A clear trend is observed in the relative abundance of $\mathrm{CH}_{3} \mathrm{OH}, \mathrm{CH}_{3} \mathrm{CN}$, and $\mathrm{C}_{2} \mathrm{H}_{5} \mathrm{CN}$ with respect to $\mathrm{H}_{2} \mathrm{CO}$ as a function of the stellar luminosity (Fig. 7a). The abundance of all these molecules increases by a factor of $\sim 10$ from NGC 1333-IRAS 4A ( $L=$ $\left.14 L_{\odot}\right)$ to OMC1 $\left(L=10^{4} L_{\odot}\right)$. This trend does not present significant differences between $\mathrm{O}-$ and $\mathrm{N}$-bearing molecules. Contrary to these molecules, the $[\mathrm{HCOOH}] /\left[\mathrm{H}_{2} \mathrm{CO}\right]$ ratio does not present any systematic behavior.

In Fig. 7b we plot the abundances of the same molecules normalized to $\mathrm{CH}_{3} \mathrm{OH}$. Different behaviors are found for the different molecules. The $\left[\mathrm{CH}_{3} \mathrm{CN}\right] /\left[\mathrm{CH}_{3} \mathrm{OH}\right]$ ratio does not present any systematic trend with the protostellar luminosity, although the dispersion in the values is quite high. The same remains true for $\mathrm{C}_{2} \mathrm{H}_{5} \mathrm{CN}$ and probably for $\mathrm{C}_{2} \mathrm{H}_{5} \mathrm{OH}$, though we only have 3 points for $\mathrm{C}_{2} \mathrm{H}_{5} \mathrm{OH}$. This suggests that the ratio between $\mathrm{O}$ - and $\mathrm{N}$-bearing complex molecules does not depend strongly on the protostellar luminosity, contrary to expectations from theoretical models. However, the relative abundance of $\mathrm{HCOOH}$ seems to decrease by 2 orders of magnitude from NGC 1333-IRAS4 A to G327.3. Thus, the relative abundances of $\mathrm{HCOOH}$ to those of $\mathrm{CH}_{3} \mathrm{OH}, \mathrm{CH}_{3} \mathrm{CN}, \mathrm{C}_{2} \mathrm{H}_{5} \mathrm{CN}$, and $\mathrm{C}_{2} \mathrm{H}_{5} \mathrm{OH}$ seem to decrease with the protostellar luminosity.

We propose that the increase in $\mathrm{CH}_{3} \mathrm{OH} / \mathrm{H}_{2} \mathrm{CO}$ (as well as $\left.\mathrm{CH}_{3} \mathrm{OH} / \mathrm{HCOOH}\right), \mathrm{CH}_{3} \mathrm{CN} / \mathrm{H}_{2} \mathrm{CO}$, and $\mathrm{C}_{2} \mathrm{H}_{5} \mathrm{CN} / \mathrm{H}_{2} \mathrm{CO}$ with

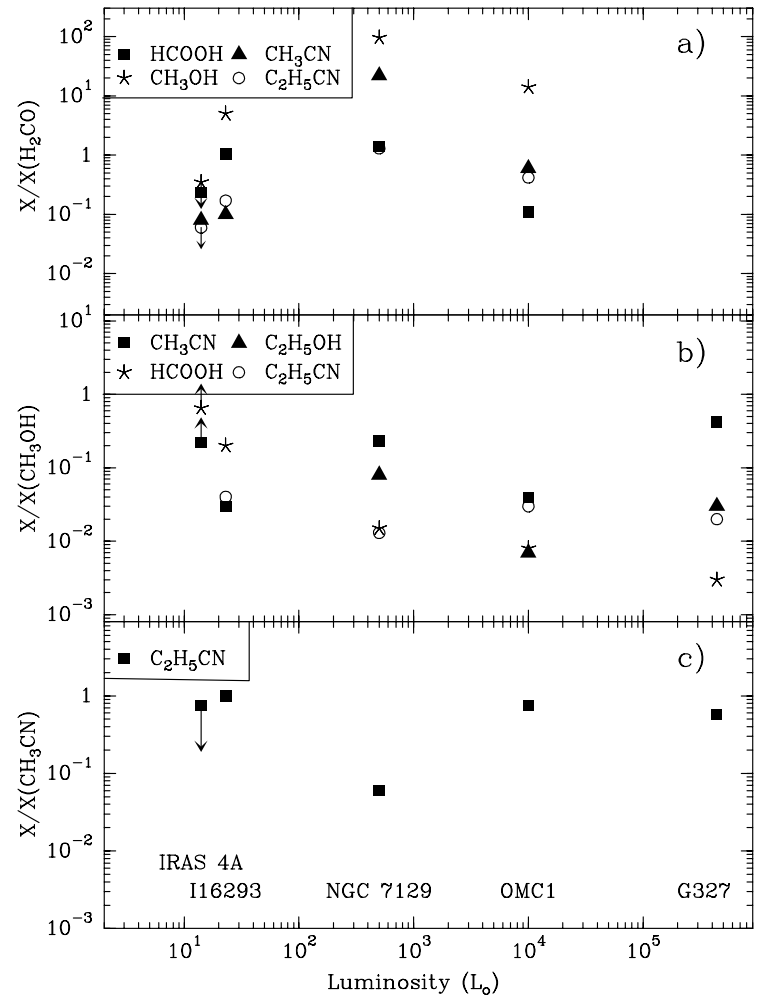

Fig. 7. Relative abundances of the complex O- and N-bearing molecules as a function of the protostellar luminosity for the objects listed in Table 3.

stellar luminosity is most likely due to differences in the grain mantle composition between low and massive star-forming regions. Indeed, differences in the initial conditions, in particular gas density and dust temperature, are known to strongly affect surface chemistry. For example, Caselli et al. (1993) found that complex N-bearing species are more easily formed if the dust grain temperature $\left(T_{\text {dust }}\right)$ is about $40 \mathrm{~K}$, during the collapse phase. Although such temperature can be found in a significant fraction of a collapsing massive clump surrounding a massive young stellar object (Fontani et al. 2002), this is not the case for low mass cores. Therefore, the observed trend (at least for $\mathrm{N}$-bearing species) is consistent with theoretical predictions. But Caselli et al. (1993) also found that at $T_{\text {dust }} \sim 40 \mathrm{~K}$, methanol is not efficiently formed on the surface because it requires the volatile $\mathrm{H}$ to stick on the grain surface (see also Charnley et al. 1992; van der Tak et al. 2000). Thus, the observed $\mathrm{CH}_{3} \mathrm{OH} / \mathrm{H}_{2} \mathrm{CO}$ increase with stellar luminosity is not well understood, unless another surface formation mechanism for methanol (besides the $\mathrm{CO}$ hydrogenation) becomes important in the warmer high mass clumps, e.g. $\mathrm{OH}+\mathrm{CH}_{3}$ (see also discussion in Pontoppidan et al. 2003). However, this conclusion is only based on 4 sources and has to be confirmed with a larger and more complete sample. Observational bias, such as the different spatial scale for the different objects, can contribute to this trend if the spatial extension of the emission of the observed molecules is different.

In Fig. 7c, we compare only the N-bearing molecules. The $\left[\mathrm{C}_{2} \mathrm{H}_{5} \mathrm{CN}\right] /\left[\mathrm{CH}_{3} \mathrm{CN}\right]$ ratio remains quite constant with a dispersion of about a factor $<10$ between all the sources. This 
uniform $\left[\mathrm{C}_{2} \mathrm{H}_{5} \mathrm{CN}\right] /\left[\mathrm{CH}_{3} \mathrm{CN}\right]$ ratio suggests that the chemistry of both compounds is linked. Indeed, these two molecules have a common parent species: $\mathrm{C}_{2} \mathrm{~N}$ (see e.g. Fig. 7 in Caselli et al. 1993)

\section{Conclusions: the chemistry of the IM hot core NGC 7129-FIRS 2}

In this paper we present the first detection of an intermediatemass hot core. Our interferometric observations towards FIRS 2 allow us to estimate a size of $650 \times 900 \mathrm{AU}$ and a mass of $2 M_{\odot}$ for the hot core associated with this IM object. The dimensions and mass of this IM hot core are intermediate between those measured in hot corinos $\left(r \sim 150 \mathrm{AU}, M<1 M_{\odot}\right)$ and massive stars $\left(r \sim 3000 \mathrm{AU}, M>10 M_{\odot}\right)$, and consequently one expects to find a differentiated chemistry in it.

Our interferometric observations of $\mathrm{CH}_{3} \mathrm{CN}, \mathrm{CH}_{3} \mathrm{OH}$, $\mathrm{D}_{2} \mathrm{CO}$, and $\mathrm{N}_{2} \mathrm{D}^{+}$provides information on the structure and chemistry of this object. While the $\mathrm{CH}_{3} \mathrm{CN}$ emission arises mainly in the hot core, the $\mathrm{CH}_{3} \mathrm{OH}$ emission has a component associated with the bipolar outflow. The $\mathrm{CH}_{3} \mathrm{CN}$ abundance is $\sim 7.0 \times 10^{-9}$ in the hot core, which is about 3 orders of magnitude larger than in the cool envelope. The methanol abundance is enhanced $\left(>3 \times 10^{-8}\right.$-a few $\left.10^{-7}\right)$ in the hot core and the outflow components. However, the rotation temperature is higher in the hot core than in the outflow, revealing very different physical conditions and probably a different $\mathrm{CH}_{3} \mathrm{OH}$ desorption mechanism for the two components.

The doubly deuterated formaldehyde also presents enhanced abundances in the hot core. We estimate a $\left[\mathrm{D}_{2} \mathrm{CO}\right] /\left[\mathrm{H}_{2} \mathrm{CO}\right] \sim 0.14$, which is 4 orders of magnitude larger than the cosmic D abundance and similar to those found in pre-stellar clumps and low-mass protostars. The enhanced $\left[\mathrm{D}_{2} \mathrm{CO}\right] /\left[\mathrm{H}_{2} \mathrm{CO}\right]$ abundance in the hot core suggests that grain surface chemistry is responsible of the deuteration process of $\mathrm{H}_{2} \mathrm{CO}$. In contrast to the high deuteration degree of formaldehyde, we did not detect $\mathrm{N}_{2} \mathrm{D}^{+}$in the hot core. This is consistent with the chemical scheme in which, while the deuteration of $\mathrm{H}_{2} \mathrm{CO}$ requires surface chemistry, the deuteration of $\mathrm{N}_{2} \mathrm{H}^{+}$is a gas-phase process.

A large number of molecular lines have been detected in our interferometric spectra towards FIRS 2. Most of these lines are identified as belonging to deuterated $\left(\mathrm{D}_{2} \mathrm{CO}, \mathrm{c}-\mathrm{C}_{3} \mathrm{D}\right.$ and $\left.\mathrm{c}-\mathrm{C}_{3} \mathrm{HD}\right)$, sulphuretted $\left({ }^{13} \mathrm{CS}\right.$, OCS), and complex O-/Nbearing species $\left(\mathrm{HCOOH}, \mathrm{C}_{2} \mathrm{H}_{5} \mathrm{OH}, \mathrm{C}_{2} \mathrm{H}_{5} \mathrm{CN}\right)$. Deuterated species, whose deuteration requires surface chemistry such as $\mathrm{D}_{2} \mathrm{CO}$, present enhanced abundances in the warm regions associated with low-mass protostars. Loinard et al. (2003) searched for the doubly deuterated form of formaldehyde $\left(\mathrm{D}_{2} \mathrm{CO}\right)$ in a large sample of young stellar objects. $\mathrm{D}_{2} \mathrm{CO}$ was detected in all low-mass protostars with $\left[\mathrm{D}_{2} \mathrm{CO}\right] /\left[\mathrm{H}_{2} \mathrm{CO}\right]$ ratios of $2-40 \%$. On the other hand, no detection was obtained towards more massive protostars, where $\left[\mathrm{D}_{2} \mathrm{CO}\right]\left[\mathrm{H}_{2} \mathrm{CO}\right]<0.5 \%$. This is consistent with the results reported by Turner (1990), who detected $\mathrm{D}_{2} \mathrm{CO}$ in Orion and measured $\left[\mathrm{D}_{2} \mathrm{CO}\right] /\left[\mathrm{H}_{2} \mathrm{CO}\right] \sim 0.003$. If the hot cores associated with massive stars are older or significantly denser than those surrounding low-mass objects, gas phase chemistry could have had time to re-set the deuterium fractionation to values close to the cosmic $\mathrm{D} / \mathrm{H}$ ratio. An alternative explanation is that the gas temperature of the material accreting high mass protostars is higher than $30 \mathrm{~K}$ (Fontani et al. 2002), so that the deuterium fractionation efficiency in the gas phase is strongly reduced already before the hot core phase.

The sulphuretted and complex compounds are characteristic of hot cores in both the low-mass and the high-mass regimes. FIRS 2 is the first IM hot core detected thus far and presents a unique opportunity to study the link between the chemistry of hot corinos and massive hot cores. We have compared the abundances of complex molecules in FIRS 2 with those in hot corinos and the massive hot cores OMC1 and G327.3-0.6. Contrary to model predictions, we did not detect any dependence of the O-/N-complex molecules ratio on the protostellar luminosity. However, we detected differences between the behavior of the O-bearing species with the stellar luminosity. While $\mathrm{H}_{2} \mathrm{CO}$ and $\mathrm{HCOOH}$ are more abundant in low luminosity sources, $\mathrm{CH}_{3} \mathrm{OH}$ seems to be more abundant in massive objects. We propose that this could be due to a different mantle composition in the two kinds of region, caused by different physical conditions (mainly gas density and dust temperature) during the pre-stellar and accretion phase. However, this could also be due to other factors, such as the different spatial scale of the observations or a possible contribution of the shocked gas associated with the bipolar outflow to the emission of these molecules. Differences in the hot core ages and/or cloud initial conditions could also produce these differences. The detection and detailed study of more intermediate-mass hot cores are necessary to reach firm conclusions.

Acknowledgements. This work was partially supported by the Spanish DGICYT under grant AYA2003-07584 and Spanish DGI/SEPCT under grant ESP2003-04957. PC acknowledges support from the MIUR grant "Dust and molecules in astrophysical environments". We are also grateful to the anonymous referee for his/her fruitful comments.

\section{References}

Bacmann, A., Lefloch, B., Ceccarelli, C., et al. 2003, ApJ, 585, L55

Bachiller, R., \& Perez Gutierrez, M. 1997, ApJ, 487, L93

Bergin, E. A., Alves, J., Huard, T., \& Lada, C. J. 2002, ApJ, 570, L101

Bottinelli, S., Ceccarelli, C., Lefloch, B., et al. 2004, ApJ, 615, L354

Brown, P. D., Charnley, S. B., \& Millar, T. J. 1988, MNRAS, 231, 409

Butner, H. M., Lada, E. A., \& Loren, R. B. 1995, ApJ, 448, 207

Caselli, P., Hasegawa, T. I., \& Herbst, E. 1993, ApJ, 408, 548

Caselli, P., Walmsley, C. M., Tafalla, M., Dore, L., \& Myers, P. C. 1999, ApJ, 523, L165

Caselli, P., Walmsley, C. M., Zucconi, A., et al. 2002a, ApJ, 565, 331

Caselli, P., Stantcheva, T., Shalabiea, O., Shematovich, V. I., \& Herbst, E. $2002 b, P \& S S, 50,1257$

Caselli, P., van der Tak, F. F. S., Ceccarelli, C., \& Bacmann, A. 2003, A\&A, 403, L37

Cazaux, S., Tielens, A. G. G. M., Ceccarelli, C., et al. 2003, ApJ, 593, L51

Ceccarelli, C., Loinard, L., Castets, A., Tielens, A. G. G. M., \& Caux, E. 2000, A\&A, 357, L9

Ceccarelli, C., Loinard, L., Castets, A., et al. 2001, A\&A, 372, 998

Ceccarelli, C., Vastel, C., Tielens, A. G. G. M., et al. 2002, A\&A, 381, L17 
Crapsi, A., Caselli, P., Walmsley, C. M., et al. 2005, ApJ, 619, 379

Charnley, S. B., Tielens, A. G. G. M., \& Millar, T. J. 1992, ApJ, 399, L71

Charnley, S. B., Tielens, A. G. G. M., \& Rodgers, S. D. 1997, ApJ, 482, L203

Fontani, F., Cesaroni, R., Caselli, P., \& Olmi, L. 2002, A\&A, 389, 603, L203

Fuente, A., Martín-Pintado, J., et al. 1998, A\&A, 334, 253

Fuente, A., Neri, R., Martín-Pintado, J., Bachiller, R., RodríguezFranco, A., \& Palla, F. 2001, A\&A, 366, 873 (Paper I)

Fuente, A., Martín-Pintado, J., Bachiller, R., Rodríguez-Franco, A., \& Palla, F. 2002, A\&A, 387, 977

Fuente, A., Rodríguez-Franco, A., Testi, L., et al. 2003, ApJ, 598, L39

Fuente, A., Rizzo, J. F., Caselli, P., Bachiller, R., \& Henkel, C. 2005, A\&A, 433, 535 (Paper II)

Gibb, E., Nummelin, A., Irvine, W. M., Whittet, D. C. B., \& Bergman, P. 2000, ApJ, 545, 309

Hollis, J. M., Jewell, P. R., Lovas, F. J., Remijan, A., \& Møllendal, H. 2004, ApJ, 610, L21

Horn, A., Møllendal, H., Sekiguchi, O., et al. 2004, ApJ, 611, 605

Kramer, C., Alves, J., Lada, C. J., et al. 1999, A\&A, 342, 257

Loinard, L., et al. 2003, SFChem 2002: Chemistry as a Diagnostic of Star Formation, proceedings of a conference held August 21-23, 2002 at University of Waterloo, Waterloo, Ontario, Canada N2L 3G1, ed. L. Charles Curry, \& Michel Fich (Ottawa Canada: NRC Press), 351

Maret, S., Ceccarelli, C., Caux, E., et al. 2004, A\&A, 416, 577

Pickett, H. M., Poynter, R. L., Cohen, E. A., et al. 1998, J. Quant. Spectrosc. \& Rad. Transfer, 60, 883
Pontoppidan, K. M., Dartois, E., van Dishoeck, E. F., Thi, W.-F., \& d'Hendecourt, L. 2003, A\&A, 404, L17

Rodgers, \& Charnley 2001, ApJ, 546, 324

Roberts, H., Herbst, E., \& Millar, T. J. 2003, ApJ, 591, L41

Rodríguez, L. F., Loinard, L., D’Alessio, P., Wilner, D. J., \& Ho, P. T. P. 2005, ApJ, 621, L133

Schoier, F. L., Jorgensen, J. K., van Dishoeck, E. F., \& Blake, G. A. 2002, A\&A, 390, 1001

Stantcheva, T., \& Herbst, E. 2003, MNRAS, 340, 983

Sutton, E. C., Peng, R., Danchi, W. C., et al. 1995, ApJS, 97, 455

Tafalla, M., Myers, P. C., Caselli, P., Walmsley, C. M., \& Comito, C. 2002, ApJ, 569, 815

Tielens, A. G. G. M. 1983, A\&A, 119, 177

Turner, B. E. 1990, ApJ, 362, L29

van der Tak, F. F. S., van Dishoeck, E. F., \& Caselli, P. 2000, A\&A, 361,327

Vastel, C., Phillips, T. G., \& Yoshida, H. 2004, ApJ, 606, L127

Viti, S., Collings, M. P., Dever, J. W., McCoustra, M. R. S., \& Williams, D. A. 2004, MNRAS, 354, 1141

Wakelam, V., Caselli, P., Ceccarelli, C., Herbst, E., \& Castets, A. 2004, A\&A, 422, 159

Walmsley, C. M., Flower, D. R., \& Pineau des Forêts, G. 2004, A\&A, 418, 1035

Watson, W. D. 1974, ApJ, 188, 35

Willacy, K., Langer, W. D., \& Velusamy, T. 1998, ApJ, 507, L171

Williams, J. P., Bergin, E. A., Caselli, P., Myers, P. C., \& Plume, R. 1998, ApJ, 503, 689

Wyrowski, F., Schilke, P., \& Walmsley, C. M. 1999, A\&A, 341, 882 